\title{
Nonassociative geometry and twist deformations in non-geometric string theory
}

\author{
Dionysios Mylonas, Peter Schupp and Richard J. Szabo* \\ Heriot-Watt University, Edinburgh, U.K. \\ E-mail: dm281@hw.ac.uk, R.J.Szabo@hw.ac.uk \\ Jacobs University Bremen, Germany \\ Email: p.schuppejacobs-university.de
}

\begin{abstract}
We describe nonassociative deformations of geometry probed by closed strings in non-geometric flux compactifications of string theory. We show that these non-geometric backgrounds can be geometrised through the dynamics of open membranes whose boundaries propagate in the phase space of the target space compactification, equiped with a twisted Poisson structure. The effective membrane target space is determined by the standard Courant algebroid over the target space twisted by an abelian gerbe in momentum space. Quantization of the membrane sigma-model leads to a proper quantization of the non-geometric background, which we relate to Kontsevich's formalism of global deformation quantization that constructs a noncommutative nonassociative star product on phase space. We construct Seiberg-Witten type maps between associative and nonassociative backgrounds, and show how they may realise a nonassociative deformation of gravity. We also explain how this approach is related to the quantization of certain Lie 2-algebras canonically associated to the twisted Courant algebroid, and cochain twist quantization using suitable quasi-Hopf algebras of symmetries in the phase space description of $R$-space which constructs a Drinfel'd twist with non-trivial 3-cocycle. We illustrate and apply our formalism to present a consistent phase space formulation of nonassociative quantum mechanics.
\end{abstract}

Report number: EMPG-14-5

Third International Satellite Conference on Mathematical Methods in Physics - ICMP 2013

21-26 October, 2013

Londrina, PR, Brazil

${ }^{*}$ Speaker. 


\section{Contents}

1. String geometry 2

2. Non-geometric string backgrounds $\quad 4$

2.1 Non-geometric flux compactification 4

$\begin{array}{ll}2.2 & \text { Magnetic backgrounds in quantum mechanics }\end{array}$

2.3 Geometry of $n$-algebras $\quad 7$

$\begin{array}{lll}2.4 & \text { Closed string noncommutative and nonassociative geometry } & 8\end{array}$

3. Geometrization of non-geometry 11

3.1 Generalized, doubled and phase space geometry 11

$3.2 n$-algebroids and AKSZ sigma-models $\quad 12$

$\begin{array}{lll}3.3 & \text { Sigma-models for geometric fluxes } & 14\end{array}$

$\begin{array}{lll}3.4 & \text { Sigma-models for non-geometric fluxes } & 15\end{array}$

4. Quantization of $\boldsymbol{R}$-flux string vacua $\quad 16$

4.1 Path integral quantization 16

$\begin{array}{lll}4.2 & \text { Seiberg-Witten maps and noncommutative gerbes } & 19\end{array}$

$\begin{array}{ll}4.3 & 3 \text {-cocycles and categorified Weyl quantization } \\ 4.4 & 20\end{array}$

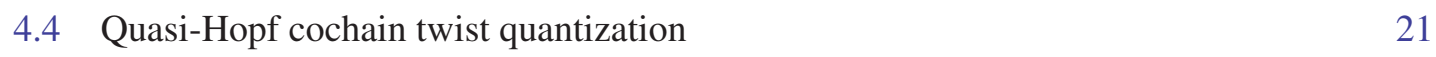

4.5 Quantum mechanics with a 3-cocycle 23

\section{String geometry}

Strings see geometry in different ways than point particles do because of their extended nature. This feature has led to many novel examples of string geometry which involve modifications, sometimes radical, of standard geometric structures. One of the first examples of such string symmetries was T-duality, which due to string winding modes implies that large and small compact directions are indistinguishable in string theory. Together with related phenomena such as mirror symmetry, these predictions from string theory have opened up many unexpected developments in geometry. In many such cases string theory is usually studied in regimes whereby a geometric description is available. However, via T-duality string theory also admits non-geometric backgrounds as consistent solutions [23].

It has been long hoped that closed strings, and also other degrees of freedom in string theory, provide good probes of Planck scale quantum geometry where the classical notions of general relativity break down. In fact, a simple argument for the semi-classical quantization of gravity suggests that spacetime coordinates themselves should be subjected to an uncertainty principle $\Delta x \geq \ell_{P}$; here $\ell_{P}$ is some suitable fundamental length scale such as the Planck scale or the string 
length. This requirement ensures that one cannot localize events in spacetime so as to produce strong gravitational fields that hide the events to distant observers. In this article we shall review how such spacetime uncertainties are related to noncommutative spacetime structures and to nongeometry in string theory. We describe how a complementary target space approach to quantum gravity based on noncommutative geometry is related to certain non-geometric generalizations of the target spacetime in string theory.

A precise realisation of noncommutative geometry in string theory first appeared in the dynamics of the open string sector. It was realised some time ago that D-branes in constant background Neveu-Schwarz $B$-fields provide concrete dynamical realisations of noncommutative spaces [19, $61,62]$. The low-energy dynamics of this system is described by a noncommutative gauge theory on the D-brane worldvolume, together with Seiberg-Witten maps which relate them to ordinary (commutative) gauge theories. These developments have led to a flurry of investigation over the past 15 years into the structures and properties of these noncommutative field theories; see e.g. $[25,64]$ for early pedagogical reviews on the subject. The key feature of these realisations is that they involve a low-energy regime of string theory which decouples all massive open string states and all closed string modes while still retaining the effects of noncommutativity. The emergent noncommutative geometry in this limit is completely analogous to that of electron coordinates in a constant magnetic background in the lowest Landau level (see e.g. [65]).

Analogous structures can also arise in the dynamics of the closed string sector as a result of the intertwining of momentum and winding modes in non-geometric backgrounds. To understand this point, let us recall how noncommutativity in the open string sector arises following [62]. In the Seiberg-Witten scaling limit which decouples the bulk closed string modes from the open string boundary degrees of freedom, the two-point function for string field insertions on the boundary of a disk in tree-level open string perturbation theory depends only on the relative ordering of the insertion points, and not on their actual positions. This means that the two-point function is a well-defined target space entity that is independent of the worldsheet coordinates. It leads to a noncommutative 2-bracket between string coordinates, with the deformation provided by a bivector which is determined by the inverse of the constant two-form $B$-field. Quantization of this 2-bracket yields a star product of fields on D-brane worldvolumes and noncommutative gauge theory as a deformation of the low-energy effective field theory in the open string sector.

The natural question of how analogous structures can arise in the closed string sector has remained somewhat unclear until the recent works [11, 48, 14]. At tree-level in closed string perturbation theory, the two-point function on a sphere depends explicitly on the worldsheet coordinates in the low-energy limit, and so is not a bonafide target space quantity. On the other hand, the threepoint function depends only on the relative orientation of the three insertion points on the sphere, and hence a 3-bracket structure emerges on target space. It is natural to identify this 3-bracket as a measure of nonassociativity of the closed string coordinates, and in this case the deformation is provided by a trivector induced by a non-geometric $R$-flux which is T-dual to the constant threeform $H$-flux of a Neveu-Schwarz $B$-field. Quantization of this 3-bracket structure is anticipated to lead to closed string nonassociative gravity as a deformation of the low-energy effective field theory in the closed string sector.

In contrast to open string noncommutativity, which can arise in on-shell string scattering amplitudes because only the cyclic ordering of vertex operators is conformally invariant, the nonasso- 
ciativity observed here is a truly off-shell phenomenon. Once momentum conservation in tachyon scattering amplitudes is taken into account, all traces of nonassociativity disappear and the usual crossing symmetry of correlation functions in two-dimensional conformal field theory is recovered. The appearence of nonassociativity should be regarded as a feature whose consistency induces constraints, such as flux quantization, and teaches us something about the nature of non-geometric string theory. In the following we shall tackle this nonassociative deformation of geometry head on and describe techniques for describing its proper quantization, which should be regarded as a type of short distance spacetime quantization which is not seen by strings due to their finite intrinsic length and resolution; in this sense these non-geometric quantum geometries can arise as consistent string backgrounds. In particular, we shall show how nonassociativity can be accounted for via the derivation of dynamical star-products, via a categorified version of Weyl quantization, and through quasi-Hopf cochain twist quantization techniques. Our quantization obeys the requisite cyclicity requirements of two-dimensional conformal field theory, and can be used to formulate a consistent nonassociative version of quantum mechanics. We will describe several analogues of the Seiberg-Witten map interpolating between associative and nonassociative theories, particularly in the framework of a nonassociative theory of gravity, and in the context of extending these structures to non-constant backgrounds.

The appearence of nonassociative geometry in string theory and of systematic noncommutative deformations of gravity is not new. Nonassociative gauge theories arise naturally in the open string sector when D-branes are placed in a non-constant $B$-field background; the nonassociative deformation in this case is controlled by the three-form $H$-flux $H=\mathrm{d} B \neq 0$ through Kontsevich's deformation quantization of $H$-twisted Poisson structures which is reproduced by the correlation functions of open string tachyon vertex operators [21, 33, 31]. These open string nonassociative spaces are described locally by associative algebras and noncommutative gerbes [7] with characteristic class $[H]$ via a patching of the twisted Poisson manifold. Cyclicity appears and nonassociativity disappears in on-shell tachyon scattering amplitudes by using the Dirac-Born-Infeld field equations on the D-brane [31,32]. On the other hand, noncommutative gravity has been formulated within the cocycle twist deformation framework of [8] which gives a systematic formulation of differential geometry and general relativity on noncommutative spacetime. However, it is argued by [3] that the twisted diffeomorphisms underlying the symmetries of these associative noncommutative deformations do not arise as physical symmetries of string theory, and that the low-energy effective field theory contains terms which cannot be accounted for by a noncommutative deformation of gravity. Although all of these structures will appear in the following, as long as we are looking for (nonassociative) quantizations of gravity within the realm of string theory, the mechanisms and features of the theory we develop in the following will be rather different in nature.

\section{Non-geometric string backgrounds}

\subsection{Non-geometric flux compactification}

Compactifications of string theory are required to relate them to observable phenomenology and cosmology. Flux compactifications include $p$-form fluxes along the compact directions; they 
stabilize moduli and can lead to generalized topological as well as geometric structures wherein open neighbourhoods are patched together by string symmetries. In particular, by demanding Tduality covariance, Neveu-Schwarz $H$-fluxes naturally lead to non-geometric fluxes. While the standard geometric flux compactifications do not give all four-dimensional supergravity theories, non-geometric fluxes enable more gaugings and avoid many no-go theorems. In this section we describe the emergence of non-geometry in flux compactifications of string theory, and how nongeometric $Q$-fluxes lead to closed string noncommutativity.

The prototypical string background is provided by the three-torus $T^{3}$ with constant $H$-flux and dilaton field, which by application of the Büscher rules gives rise to geometric and non-geometric fluxes via the T-duality chain $[37,63]$

$$
H_{i j k} \stackrel{T_{i}}{\longrightarrow} f^{i}{ }_{j k} \stackrel{T_{j}}{\longrightarrow} Q^{i j}{\stackrel{T_{k}}{\longrightarrow}}^{i j k} .
$$

Here $T_{i}, i=1,2,3$ denotes a T-duality transformation along the $i$-th cycle of $T^{3}$, which in each step maps the flux to a new flux with a raised index; geometrically this means that a given differential form component is dualised to a vector field component. Let us run through the geometric and non-geometric interpretations of each duality frame in the chain (2.1).

The first member of the T-duality chain is the original flat torus $T^{3}$ with $H$-flux $H=\mathrm{d} B$. Abelian fluxes in string theory obey analogues of the Dirac quantization condition (see e.g. [66]), and hence the three-form determines a cohomology class $[H] \in \mathrm{H}^{3}\left(T^{3} ; \mathbb{Z}\right)=\mathbb{Z}$ which is the characteristic class of a gerbe. Such a gerbe lies within the realm of what we shall consider as a geometric background.

The next member of the chain involves a metric flux $f$, which determines a torsion in the geometry through the Cartan-Maurer equations

$$
\mathrm{d} e^{i}=-\frac{1}{2} f^{i}{ }_{j k} e^{j} \wedge e^{k}
$$

for one-forms $e^{i}$ dual to a local frame of vector fields $e_{i}$ with $\left[e_{i}, e_{j}\right]=f^{k}{ }_{i j} e_{k}$. In this T-duality frame the $B$-field vanishes and the geometry is that of a twisted torus or Heisenberg nilmanifold, which is a circle bundle of degree $[H]$ over a two-torus $T^{2}[60,45]$. Hence this is still a geometric frame.

The situation becomes more interesting at the next member of (2.1) which involves nongeometric $Q$-flux; closed strings in $Q$-space can be locally modeled as a $T^{2}$ bundle over a circle $S^{1}$. In this case the closed string momentum and winding modes become entangled, and the background is called a $T$-fold as the transition functions between local charts now involve stringy Tduality transformations [30, 22, 37]. In this instance the metric and $B$-field are well-defined locally but not globally; in particular, the $T^{2}$-fibre is glued back to itself by an $\operatorname{SL}(2, \mathbb{Z})$ transformation as one winds around the base $S^{1}$. In $[51,28,16]$ it is argued that this non-geometry at the topological level can be regarded globally as a fibration over $S^{1}$ by noncommutative two-tori $T_{\theta}^{2}$ with the commutation relations $\left[x^{i}, x^{j}\right]=\mathrm{i} Q^{i j}{ }_{k} x^{k}$ among local coordinates. Here $\theta^{i j}(x)=Q^{i j}{ }_{k} x^{k}$ determine a field of local noncommutativity parameters parametrized by the coordinates of the base $S^{1}$; this bivector is naturally dual to the $B$-field which is a potential for the original $H$-flux in the T-duality chain. These topological arguments are based in the open string sector in the sense that they exploit the realisation of T-duality in the twisted $\mathrm{K}$-theory of certain $C^{*}$-algebras; in particular, in the classical limit $\theta=0$ they realise a geometric space only up to Morita equivalence. Later on we shall 
see how to regard this identification more concretely and physically in the context of closed strings which wind in the non-geometric background.

The final member of the T-duality chain (2.1) involves the non-geometric $R$-flux and leads to a background which is not even locally geometric [63]. One may object to the existence of this background because a $B$-field which sources a constant non-zero $H$-flux is non-constant, hence the final direction is not a Killing isometry of the background and the standard Büscher rules cannot be applied to the $Q$-flux background. However, the prescription is completely well-defined at the level of worldsheet conformal field theory, and the final T-duality transformation is performed by flipping the sign of the corresponding right-moving closed string coordinate; the failure of the Büscher rules simply reflects the absence of local geometric structures. It is argued by $[15,26]$ that the globalisation of this non-geometry is a (topological) nonassociative three-torus, regarded as a fibration over a point in which the local fibre coordinates obey a 3-bracket relation of the form $\left[x^{i}, x^{j}, x^{k}\right]=R^{i j k}$; in this setting nonassociativity is realised in terms of twisted convolution products in a Busby-Smith convolution $C^{*}$-algebra, which again reduces in the classical limit to a geometric setting only up to Morita equivalence. Later on we shall find more concrete physical realisations of such nonassociative spaces in the context of closed strings which propagate in the non-geometric background. We are only beginning to understand the (non-)geometry of $R$-flux backgrounds in terms of new types of spatial noncommutative and nonassociative structures.

\subsection{Magnetic backgrounds in quantum mechanics}

To help understand the meaning of a nonassociative geometry, we shall now describe a simple magnetic field analog of nonassociativity in the ordinary quantum mechanics of point particles. This example illustrates that in fact nonassociativity arises naturally in the wild, and how it should be interpreted in the context of closed string non-geometric backgrounds. For this, let us start by recalling that the noncommutative deformations of $\mathrm{D}$-brane worldvolumes induced by open strings in constant $B$-field backgrounds in the Seiberg-Witten scaling limit have a natural analog in the physics of the Landau problem (see e.g. [65]). For charged particles constrained to move in two dimensions under the influence of a perpendicularly applied constant magnetic field $\boldsymbol{B}=B \hat{z}$, the strong field limit projects the system to the lowest Landau level and the effective Lagrangian is of first order in time derivatives of the particle coordinates $(x, y)$; canonical quantization thereby gives a noncommutative position space with relations $[x, y]=\mathrm{i} \theta$, where the noncommutativity parameter $\theta$ is proportional to $B^{-1}$.

A more general magnetic field analog was proposed some time ago by Jackiw [40]. Charged particles in three dimensions experience a magnetic field $\boldsymbol{B}$ (with sources) via the Lorentz force law $\boldsymbol{\pi}=\frac{e}{m} \boldsymbol{\pi} \times \boldsymbol{B}$ for the physical (gauge covariant) momentum $\boldsymbol{\pi}$. The Hamiltonian $\mathscr{H}=\frac{1}{2 m} \boldsymbol{\pi}^{2}$ generates the Lorentz force $\boldsymbol{\pi}=\mathrm{i}[\mathscr{H}, \boldsymbol{\pi}]$ only for a noncommutative momentum space described by the phase space commutation relations

$$
\left[x^{i}, x^{j}\right]=0 \quad, \quad\left[x^{i}, \pi_{j}\right]=\mathrm{i} \hbar \delta^{i}{ }_{j} \quad, \quad\left[\pi_{i}, \pi_{j}\right]=\mathrm{i} \hbar e \varepsilon_{i j k} B^{k} .
$$

In a magnetic background translation invariance is lost, but there is still a gauge symmetry under the magnetic translations $U(\boldsymbol{a})=\mathrm{e}^{\frac{\mathrm{i}}{\hbar} \boldsymbol{a} \cdot \boldsymbol{\pi}}$. It follows from the commutation relations and the BakerCampbell-Hausdorff formula that they do not commute,

$$
U\left(\boldsymbol{a}_{1}\right) U\left(\boldsymbol{a}_{2}\right)=\mathrm{e}^{-\frac{\mathrm{i} e}{\hbar} \Phi_{a_{1}, a_{2}}} U\left(\boldsymbol{a}_{1}+\boldsymbol{a}_{2}\right),
$$


where $\Phi_{\boldsymbol{a}_{1}, \boldsymbol{a}_{2}}=\frac{1}{2}\left(\boldsymbol{a}_{1} \times \boldsymbol{a}_{2}\right) \cdot \boldsymbol{B}$ is the magnetic flux through the (infinitesimal) triangle $\left\langle\boldsymbol{a}_{1}, \boldsymbol{a}_{2}\right\rangle$ spanned by the two vectors. This is simply the well-known result that the wavefunctions of a particle in a magnetic background only carry a projective representation of the translation group; in particular, the projective phase is a 2-cocycle of the abelian group of translations.

More dramatically, nonassociativity generically arises through the violation of the Jacobi identity, as can be seen by computing the Jacobiator

$$
\left[\pi_{i}, \pi_{j}, \pi_{k}\right]:=\left[\pi_{i},\left[\pi_{j}, \pi_{k}\right]\right]+\left[\pi_{j},\left[\pi_{k}, \pi_{i}\right]\right]+\left[\pi_{k},\left[\pi_{i}, \pi_{j}\right]\right]=-e \hbar^{2} \varepsilon_{i j k} \nabla \cdot \boldsymbol{B}=:-e \hbar^{2} H_{i j k} .
$$

As a consequence, the magnetic translations do not associate and have an associator given by

$$
\left(U\left(\boldsymbol{a}_{1}\right) U\left(\boldsymbol{a}_{2}\right)\right) U\left(\boldsymbol{a}_{3}\right)=\mathrm{e}^{-\frac{\mathrm{i} e}{\hbar} \Phi_{\boldsymbol{a}_{1}, a_{2}, \boldsymbol{a}_{3}} U\left(\boldsymbol{a}_{1}\right)\left(U\left(\boldsymbol{a}_{2}\right) U\left(\boldsymbol{a}_{3}\right)\right)}
$$

where $\Phi_{a_{1}, a_{2}, a_{3}}=\frac{1}{6}\left(\left(\boldsymbol{a}_{1} \times \boldsymbol{a}_{2}\right) \cdot \boldsymbol{a}_{3}\right) \nabla \cdot \boldsymbol{B}$ is the magnetic flux through the (infinitesimal) tetrahedron $\left\langle\boldsymbol{a}_{1}, \boldsymbol{a}_{2}, \boldsymbol{a}_{3}\right\rangle$ spanned by the three vectors. Here the projective phase is a 3 -cocycle of the abelian group of translations.

There are now two cases to consider. If we demand that the magnetic field satisfies Maxwell's equations, then $\nabla \cdot \boldsymbol{B}=0$; in this case there are no magnetic sources, no flux, and associativity persists. Then the physical momentum operators have the standard representation $\boldsymbol{\pi}=-\mathrm{i} \hbar \nabla-e \boldsymbol{A}$ on $\mathrm{L}^{2}$-wavefunctions in terms of a vector potential $\boldsymbol{A}$ for the magnetic field; in particular, for a constant magnetic field $\boldsymbol{B}=B \hat{z}$ the commutation relations (2.2) in the strong field limit reproduce the noncommuting coordinates of the lowest Landau level [65]. On the other hand, if $\nabla \cdot \boldsymbol{B} \neq 0$ then magnetic monopoles are present, and nonassociativity of the magnetic translations persists unless

$$
\frac{e}{\hbar} \Phi_{a_{1}, a_{2}, a_{3}} \in \pi \mathbb{Z} .
$$

This is simply the Dirac quantization condition for magnetic charge; in this context it ensures the basic postulates of quantum mechanics, wherein associativity of operators is required. In this instance associativity of the global translations is satisfied, even though the infinitesimal translations still violate the Jacobi identity.

We shall see in the following that the same sort of nonassociative relations come up in the non-geometric flux models obtained via T-duality from a constant $H$-flux background, though in a "dual" sense through nonassocitivity of coordinate space rather than momentum space. The analogues of the Dirac quantization condition for $R$-flux backgrounds are described in the context of Matrix theory compactifications in [18] and of double field theory in [13]. On the other hand, a consistent nonassociative version of quantum mechanics based on the phase space quantization of the $R$-flux string model can be developed following [53] and shown rigorously to lead to minimal volume uncertainty relations $\Delta x^{i} \Delta x^{j} \Delta x^{k} \geq \frac{3}{2} \hbar^{2} R^{i j k}$ characterizing a coarse-graining of the nongeometric $R$-flux background; this is consistent with the fact that these backgrounds do not allow the introduction of point-like objects [26]. The parallels between nonassociative parabolic $R$-flux string vacua and the dynamics of charged particles in uniform magnetic charge distributions is elucidated in [9].

\subsection{Geometry of $n$-algebras}

The deformations of geometry we have been describing involve certain higher generalizations of algebraic structures, in particular certain $n$-bracket structures. We shall now explain how these extensions fit into concrete geometric frameworks, and then describe a means to quantize them. 
Let us begin by recalling that a Nambu-Poisson structure on a smooth manifold $M$ is an $n$-Lie algebra structure $\{-, \ldots,-\}: C^{\infty}(M)^{\wedge n} \rightarrow C^{\infty}(M)$, which means that it satisfies the fundamental identity

$$
\left\{f_{1}, \ldots, f_{n-1},\left\{g_{1}, \ldots, g_{n}\right\}\right\}=\left\{\left\{f_{1}, \ldots, f_{n-1}, g_{1}\right\}, \ldots, g_{n}\right\}+\cdots+\left\{g_{1}, \ldots,\left\{f_{1}, \ldots, f_{n-1}, g_{n}\right\}\right\} .
$$

In addition, it is required to obey the generalized Leibniz rule

$$
\left\{f g, h_{1}, \ldots, h_{n-1}\right\}=f\left\{g, h_{1}, \ldots, h_{n-1}\right\}+\left\{f, h_{1}, \ldots, h_{n-1}\right\} g .
$$

These properties imply that a Nambu-Poisson $n$-bracket is determined via a Nambu-Poisson $n$ vector $\Pi=\frac{1}{n !} \Pi^{i_{1} \cdots i_{n}}(x) \partial_{i_{1}} \wedge \cdots \wedge \partial_{i_{n}}$ as

$$
\left\{f_{1}, \ldots, f_{n}\right\}=\Pi\left(\mathrm{d} f_{1}, \ldots, \mathrm{d} f_{n}\right)=\Pi^{i_{1} \cdots i_{n}}(x) \partial_{i_{1}} f_{1} \cdots \partial_{i_{n}} f_{n} .
$$

In this paper our prominent example will be the flat backgrounds $M=\mathbb{R}^{3}$ or $M=T^{3}$ equiped with the Nambu-Poisson 3-bracket [54], which is defined on coordinate functions in terms of a constant trivector $R=\frac{1}{6} R^{i j k} \partial_{i} \wedge \partial_{j} \wedge \partial_{k}$ by

$$
\left\{x^{i}, x^{j}, x^{k}\right\}=R^{i j k},
$$

and extended by linearity and the generalized Leibniz rule; its quantization gives the NambuHeisenberg algebra. For further details about the quantization of generic Nambu-Poisson structures, see e.g. [24] and references therein.

We will set up a framework involving a suitable generalization of geometric quantization for these higher bracket structures; this involves the notion of multisymplectic manifolds. An $n$-plectic manifold is a manifold $M$ equiped with a closed $n+1$-form $\omega$ which obeys the nondegeneracy condition: $\omega(v,-)=0$ if and only if $v=0$. In this parlance a 1-plectic manifold is the usual symplectic manifold, while a 2-plectic manifold involves a three-form $\omega$ as in the case of geometric $H$-flux compactifications. In contrast to the symplectic case, in general there is no relation between multisymplectic and Nambu-Poisson structures. However, if $M$ has dimension $n+1$ then $\omega$ is a volume form on $M$ which can be inverted to give a Nambu-Poisson structure $\omega^{-1}$ on $M$. Such manifolds serve as multiphase spaces in Nambu mechanics, generalizing the usual Poisson phase spaces in Hamiltonian dynamics. They are the starting point for a formalism of higher quantization, which we now describe in the context of non-geometric $Q$-flux backgrounds following $[57,58]$.

\subsection{Closed string noncommutative and nonassociative geometry}

Recall that geometric quantization of a symplectic manifold $(M, \omega)$ is based on the requirement that the symplectic structure defines an integer cohomology class $[\omega] \in \mathrm{H}^{2}(M, \mathbb{Z})$ which encodes a prequantum line bundle with connection $(L, \nabla)$ whose first Chern class in Chern-Weil theory is represented by the curvature two-form $F_{\nabla}=2 \pi \mathrm{i} \omega$. Upon prescribing a suitable polarization, geometric quantization amounts to constructing a Hilbert space of sections of the line bundle $L \rightarrow M$ and a quantization map under which functions on $M$ act as operators on this Hilbert space.

In a similar fashion, a 2-plectic manifold $(M, \varpi)$ with $[\varpi] \in \mathrm{H}^{3}(M, \mathbb{Z})$ encodes a prequantum abelian gerbe with 2-connection $(\mathscr{G}, A, B)$ such that $H:=\mathrm{d} B=2 \pi \mathrm{i} \varpi$ represents the DixmierDouady class of the gerbe. It is difficult to make sense of the notions of "polarization" and a 
"Hilbert space of sections" for a gerbe, so we will instead employ the trick of $[57,58]$ which enables a proper quantization of closed string $Q$-flux backgrounds; it involves mapping 2-plectic forms to symplectic forms by transgressing the gerbe $\mathscr{G}$ to a prequantum line bundle over the loop space of the configuration manifold $M$. This approach is nicely tailored to describing closed string noncommutative geometry, as it utilizes fundamental loop variables to describe non-geometry rather than points which are not present in $R$-flux backgrounds.

We start with the geometric background $M=\mathbb{R}^{3}$ or $M=T^{3}$ endowed with a 2-plectic form given by the constant $H$-flux $H=\frac{1}{6} H_{i j k} \mathrm{~d} x^{i} \wedge \mathrm{d} x^{j} \wedge \mathrm{d} x^{k}$. Consider the correspondence

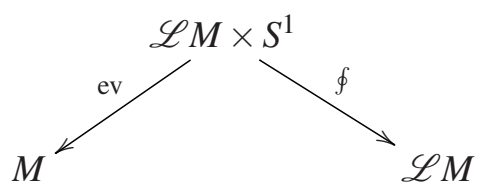

where $\mathscr{L} M=C^{\infty}\left(S^{1}, M\right)$ is the loop space of $M$ parametrizing the configuration space of closed strings in the $H$-flux background; the map ev denotes the evaluation of a loop at a point of $S^{1}$ while the map $\oint$ is integration along the $S^{1}$ fibre. The transgression map

$$
\mathscr{T}=(\oint), \circ \mathrm{ev}^{*}: \Omega^{n+1}(M) \longrightarrow \Omega^{n}(\mathscr{L} M)
$$

is defined by pulling back differential forms on $M$ via the evaluation map of (2.3) and then pushing forward along the integration over $S^{1}$. It can be expressed locally at a loop $x(\tau)$ as

$$
(\mathscr{T} \alpha)_{x}\left(v_{1}(\tau), \ldots, v_{n}(\tau)\right)=\oint \mathrm{d} \tau \alpha\left(v_{1}(\tau), \ldots, v_{n}(\tau), \dot{x}(\tau)\right)
$$

for an $n+1$-form $\alpha$ on $M$ by filling in its first $n$ slots with parametrized vector fields $v_{a}(\tau)$ on $M$ and its last slot with the velocity vector $\dot{x}(\tau)$ which is the natural tangent vector to the loop $x(\tau)$.

Via the transgression map, the background $H$-flux on $M$ yields a symplectic two-form on loop space $\mathscr{L} M$ given by

$$
\mathscr{H}:=\mathscr{T} H=\frac{1}{2} \oint \mathrm{d} \tau H_{i j k} \dot{x}^{k}(\tau) \delta x^{i}(\tau) \wedge \delta x^{j}(\tau)
$$

This two-form is always invertible and its inverse yields a Poisson bracket on $\mathscr{L} M$ with

$$
\{f, g\}_{Q}:=\oint \mathrm{d} \tau Q^{i j}{ }_{k} \frac{\dot{x}^{k}(\tau)}{|\dot{x}(\tau)|^{2}}\left(\frac{\delta}{\delta x^{i}(\tau)} f\right)\left(\frac{\delta}{\delta x^{j}(\tau)} g\right),
$$

where the dual $Q$-flux $Q^{i j}{ }_{k}$ is naturally identified with the inverse of the $H$-flux $H_{i j k}$. Quantization of this Poisson structure is rather involved and is dealt with in $[57,58]$ using an approach to quantization based on integrating the natural Lie algebroid structure on the Poisson manifold $\mathscr{L} M$ to a Lie groupoid convolution algebra. The main virtue of this approach is that it constructs the abstract algebra directly without concern about its representation on a Hilbert space, which is an appropriate arena for considerations involving nonassociativity which at the same time avoids many of the technically cumbersome constructions of geometric quantization. We will not enter into any of the technical details of this quantization procedure, which involves complicated analysis on the 
infinite-dimensional loop space $\mathscr{L} M$, except to note that the ensuing quantization map acts on the loop space coordinates by sending $x^{i}(\tau) \mapsto \hat{x}^{i}(\tau)$ with the commutation relations

$$
\left[\hat{x}^{i}(\tau), \hat{x}^{j}(\rho)\right]=\mathrm{i} \hbar Q^{i j}{ }_{k} \widehat{\dot{x}^{k}(\tau)} \delta(\tau-\rho)+\mathscr{O}\left(Q^{2}\right),
$$

where the higher order terms are described in [58]. After integration over the loop parameters $\tau, \rho \in S^{1}$, at leading order in the $Q$-flux this gives the commutation relations

$$
\left[x^{i}, x^{j}\right]=\mathrm{i} \hbar Q^{i j}{ }_{k} w^{k} \quad, \quad\left[x^{i}, w^{j}\right]=0=\left[w^{i}, w^{j}\right]
$$

where $x^{i}=\oint \mathrm{d} \tau x^{i}(\tau)$ are the closed string zero modes while $w^{i}=\oint \mathrm{d} \tau \dot{x}^{i}(\tau) \in \mathbb{Z}$ are the winding modes. This result shows why the T-fold is only locally geometric: Closed strings which wind acquire a position noncommutativity proportional to the non-geometric $Q$-flux and the winding numbers. As the relations (2.4) describe a Heisenberg Lie algebra, the resulting quantum geometry is still associative.

The same closed string noncommutativity relations were obtained by a linearized conformal field theory analysis in $[48,14,20,5]$. To linear order in the $H$-flux one can neglect the curvature backreaction of the geometry and still work on flat target space. Then one can quantize the closed string sigma-model in the constant $Q$-flux background by regarding it as a left-right asymmetric conformal field theory on a freely acting orbifold. As a closed string winds around the base circle of the local fibration $M \stackrel{T^{2}}{\longrightarrow} S^{1}$ of the T-fold, the fibre coordinates need only be periodic up to an $\operatorname{SL}(2, \mathbb{Z})$ automorphism of the torus $T^{2}$. For the parabolic flux model, which we treat throughout this paper, the monodromies of $T^{2}$ lie in a parabolic conjugacy class of $\operatorname{SL}(2, \mathbb{Z})$. The twisted boundary conditions on the fibre string fields induced by the winding numbers $w^{k}$ and by the parabolic $\operatorname{SL}(2, \mathbb{Z})$ monodromies are completely analogous to those which arise for open strings in a constant $B$-field background (see e.g. [19]); as usual, closed strings in a twisted sector of the orbifold conformal field theory can be regarded as open strings on the universal covering space of the orbifold. The resulting canonical structure and flat space mode expansion then reproduces the relations (2.4) among closed string coordinates.

This construction can be used to obtain a quantization of the $R$-flux background by applying formal T-duality, which sends the $Q$-flux $Q^{i j}{ }_{k}$ to the $R$-flux $R^{i j k}$ and the closed string winding variables $w^{k}$ to the momentum modes $p_{k}$ which are canonically conjugate to the string position coordinates $x^{i}$. Under this transformation the commutation relations (2.4) are mapped to

$$
\left[x^{i}, x^{j}\right]=\mathrm{i} \hbar R^{i j k} p_{k} \quad, \quad\left[x^{i}, p_{j}\right]=\mathrm{i} \hbar \delta^{i}{ }_{j} \quad, \quad\left[p_{i}, p_{j}\right]=0 .
$$

As noted by [49, 52], these relations arise from a twisted Poisson structure on the cotangent bundle (phase space) $T^{*} M$; in particular, closed string nonassociativity is manifested in the nonvanishing Jacobiator

$$
\left[x^{i}, x^{j}, x^{k}\right]=3 \hbar^{2} R^{i j k}
$$

exactly as it emerged in the magnetic field analog. Nonassociativity in this instance arises from the non-trivial position-momentum bracket, so that the antisymmetric brackets (2.5) define only a pre-Lie algebra. In the following we shall describe the quantization of these non-geometric backgrounds to all orders in the flux. An important ingredient will be a certain open string interpretation of closed string correlation functions, based on the observation that open strings do 
not decouple from gravity in $R$-space [26] and hence open and closed strings may become indistinguishable in these backgrounds. In [48] it is in fact argued that closed string momentum and winding modes define a sort of notion of D-brane in closed string theory. A path integral description of this closed/open string duality as a result of the asymmetric twisted sectors of the underlying worldsheet conformal field theory is given in [52].

\section{Geometrization of non-geometry}

\subsection{Generalized, doubled and phase space geometry}

Let us now describe some of the techniques proposed for casting non-geometric spaces into local and global geometric formulations. A common feature to all of these geometric descriptions is that they involve some sort of a doubling of the geometry which is precisely what makes them nongeometric: In any local description the backgrounds require these additional geometric variables (such as winding coordinates or momenta below). As transition functions of a smooth manifold $M$ of dimension $d$ are valued in the structure group of the tangent bundle, the existence of stringy $\mathrm{O}(d, d)$ transition functions leads to a notion of some sort of generalized tangent bundle of rank $2 d$.

One approach to the study of non-geometric fluxes is provided by generalised geometry (see e.g. $[28,27,29])$. The key geometric object in this description is the generalized tangent bundle

$$
C=T M \oplus T^{*} M
$$

over the target space $M$. This bundle has a natural $\mathrm{O}(d, d)$-invariant metric, and a generalized metric which encodes the usual Riemannian metric and $B$-field. An abelian subgroup of suitable $\mathrm{O}(d, d)$ transformations, called $\beta$-transforms, of sections of $C$ generate the non-geometric fluxes starting from the standard geometric description in terms of a metric, $B$-field and dilaton field, which are then expressed in terms of a bivector field $\beta=\frac{1}{2} \beta^{i j} \partial_{i} \wedge \partial_{j}$; in particular, for vanishing metric flux one has

$$
Q_{k}^{i j}=\partial_{k} \beta^{i j} \quad, \quad R=[\beta, \beta]_{S}
$$

where $[-,-]_{S}$ is the Schouten bracket which is the natural extension to multivector fields of the Lie bracket of vector fields. As this transformation is not globally defined, this leads into a nongeometric framework. The generalized tangent bundle (3.1) will play a prominent role in this paper within the guise of Courant algebroids.

Another approach to the study of non-geometric fluxes is provided by doubled geometry and double field theory $[37,38,35]$ (see e.g. [1, 10, 36] for reviews). In this approach one complements the spacetime coordinates $x^{i}$ on an equal footing with their dual coordinates $\tilde{x}_{i}$ which are canonically conjugate to the winding numbers $w^{i}$, i.e., one first doubles the geometry $x^{i} \rightarrow\left(x^{i}, \tilde{x}_{i}\right)$ and $\partial_{i} \rightarrow$ $\left(\partial_{i}, \tilde{\partial}^{i}\right)$. Doubled geometry is related to generalized geometry by dropping the winding coordinates while keeping the generalized tangent bundle (3.1). The action for double field theory is cast in these variables using the generalized metric of generalized geometry and is manifestly invariant under $\mathrm{O}(d, d)$-transformations which act by rotating the doubled coordinates. Upon implementing the projection $\tilde{\partial}^{i}=0$, one recovers the usual action of supergravity. In particular, by performing a formal T-duality transformation and a field redefinition in the double field theory action using the $\mathrm{O}(d, d)$ transforms of the generalized geometry formalism, one can in this way obtain a field 
theory on $M$ for the non-geometric fluxes [6] (see also [12]). An alternative target space perspective is described in [4] at purely the supergravity level. This theory can be reproduced from double field theory by dropping some degrees of freedom and it can be rewritten in the variables of generalized geometry. It provides a geometric description with non-geometric fluxes of some non-geometric backgrounds, demonstrating that some non-geometries can be described purely at the supergravity level without passing to more involved target space frameworks or to a worldsheet formalism.

In the following we shall review our proposal [52] for the geometrization of $R$-flux backgrounds in terms of the dynamics of a membrane sigma-model. In this approach the effective target space seen by non-geometric strings now involves a doubling of $M$ to its cotangent bundle $T^{*} M$ which is interpreted as phase space, i.e., the spacetime coordinates $x^{i}$ are complemented by their conjugate momenta $p_{i}$. In this setting, the $R$-flux is a geometric three-form on $T^{*} M$ which represents the Dixmier-Douady class of an abelian gerbe on momentum space. Because of the open/closed string duality of $R$-space, the open membranes have a double life: On the one hand their dimensional reductions are closed strings which propagate in the non-geometric flux background, while on the other hand their boundaries (in the form of worldvolume branch cuts) are open strings and the membrane sigma-model can be recast as an open string $R$-twisted Poisson sigma-model with target space $T^{*} M$ whose perturbative quantization induces a nonassociative dynamical star product of fields; the corresponding Jacobiator quantizes the closed string 3-bracket.

\section{2 n-algebroids and AKSZ sigma-models}

In the next section we shall develop the systematic quantization of the nonassociative $R$-flux backgrounds by computing suitable topological sigma-model correlation functions. A general framework to describe the sorts of sigma-models we are interested in is provided by the AKSZ construction [2], which builds Chern-Simons type action functionals in the Batalin-Vilkovisky formalism for sigma-model quantum field theories whose target space is a symplectic Lie $n$-algebroid $E \rightarrow M$. Recall that a Lie algebroid is a vector bundle $E$ over a smooth manifold $M$ together with an anchor map

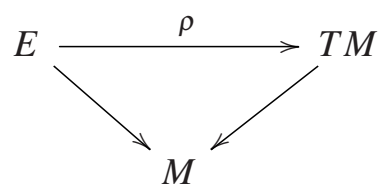

and a Lie bracket $[-,-]_{E}$ on sections $C^{\infty}(M, E)$ which is compatible with $\rho$ and the standard Lie bracket $[-,-]_{T M}$ between vector fields on the tangent bundle $T M$. A Lie algebroid simultaneously generalizes the notions of Lie algebra and tangent bundle: If $M$ is a point then a Lie algebroid is the same thing as a Lie algebra, while $E=T M$ is always a Lie algebroid with the identity anchor map. A more nontrivial example is provided by the case when $M$ is a Poisson manifold with Poisson bivector $\beta$; then the cotangent bundle $E=T^{*} M$ is a Lie algebroid with anchor map $\rho=\beta^{\sharp}: T^{*} M \rightarrow T M$ defined by $\beta^{\sharp}\left(e^{i}\right)=\beta^{i j} e_{j}$ and Lie bracket given by the Koszul bracket on oneforms: $\left[e^{i}, e^{j}\right]_{K}=\left(\partial_{k} \beta^{i j}\right) e^{k}$. Lie algebras can always be integrated to Lie groups via the exponential map, and conversely the tangent space of a Lie group at the identity is a Lie algebra. Likewise, there is a corresponding notion of Lie groupoid which is a (small) category whose object and morphism sets are smooth manifolds, and for which every morphism is invertible. However, while there is a suitable notion of differentiation of a Lie groupoid which is a Lie algebroid, not every Lie algebroid 
can be integrated to a Lie groupoid. A Lie $n$-algebroid is a categorification of a Lie algebroid which is the lowest member of the hierarchy with $n=1$; we shall not give here the general definition, as in this paper we are only interested in the cases with $n=1$ and $n=2$.

Let us begin with the case $n=1$, which is suited to the description of strings in background $B$-fields. Then the most general two-dimensional topological field theory that can be obtained from the AKSZ construction is the Poisson sigma-model [59] with target space a Lie algebroid $E \rightarrow M$ equipped with a dual section $\Theta \in C^{\infty}\left(M, \bigwedge^{2} E^{*}\right)$. Here we will only consider the case $E=T^{*} M$ with bivector field $\Theta=\frac{1}{2} \Theta^{i j}(x) \partial_{i} \wedge \partial_{j}$. Then the action of the Poisson sigma-model is given by

$$
S_{\mathrm{AKSZ}}^{(1)}=\int_{\Sigma_{2}}\left(\xi_{i} \wedge \mathrm{d} X^{i}+\frac{1}{2} \Theta^{i j}(X) \xi_{i} \wedge \xi_{j}\right)
$$

where $X: \Sigma_{2} \rightarrow M$ are embedding fields of a string worldsheet $\Sigma_{2}$ in the spacetime $M$ and $\xi \in$ $\Omega^{1}\left(\Sigma_{2}, X^{*} T^{*} M\right)$ are auxilliary one-form fields on $\Sigma_{2}$ with values in the cotangent bundle $T^{*} M$. This field theory describes the first-order form of the topological sector of a string sigma-model obtained by scaling the target space metric to zero (the Seiberg-Witten limit), and in the background of a $B$-field which is dual to the bivector $\Theta$; this can be seen by integrating out the one-form field $\xi$ explicitly, which appears quadratically in the action. Consistency of the equations of motion requires $[\Theta, \Theta]_{S}=0$ on-shell. In this case $\Theta$ defines a Poisson structure on $M$, with the vanishing Schouten bracket being equivalent to the Jacobi identity for the corresponding Poisson bracket on $C^{\infty}(M)$, and $E=T^{*} M$ is a Lie algebroid; in the general case $E$ is only a quasi-Lie algebroid, with the trivector $[\Theta, \Theta]_{S}$ controlling the violation of the Jacobi identity for the Koszul bracket. When $\Sigma_{2}$ is an open Riemann surface, the perturbative expansion of the sigma-model path integral reproduces (in both on-shell and off-shell cases) the Kontsevich formality maps for formal deformation quantization $[47,17]$.

The next member of the hierarchy of AKSZ sigma-models is the case $n=2$; a symplectic Lie 2-algebroid is the same thing as a Courant algebroid $E \rightarrow M$. A Courant algebroid is an extension of the notion of Lie algebroid which is further equiped with a fibre metric $h_{I J}=\left\langle\psi_{I}, \psi_{J}\right\rangle$, where $\psi_{I}$ is a local basis of sections for $C^{\infty}(M, E)$. The anchor matrix $\rho\left(\psi_{I}\right)=P_{I}^{i}(x) e_{i}$ and the three-form

$$
T_{I J K}(x)=\left[\psi_{I}, \psi_{J}, \psi_{K}\right]_{E}:=\frac{1}{6}\left\langle\left[\psi_{I}, \psi_{J}\right]_{E}, \psi_{K}\right\rangle+\text { cyclic }
$$

satisfy various conditions. The presence of $T_{I J K}(x)$ makes the corresponding sigma-models suited to describe dynamics in background three-tensor fluxes. There is a one-to-one correspondence between Courant algebroids and three-dimensional topological field theories obtained from the AKSZ construction, which are called Courant sigma-models $[34,39,56]$ and are described by the action

$$
S_{\mathrm{AKSZ}}^{(2)}=\int_{\Sigma_{3}}\left(\phi_{i} \wedge \mathrm{d} X^{i}+\frac{1}{2} h_{I J} \alpha^{I} \wedge \mathrm{d} \alpha^{J}-P_{I}^{i}(X) \phi_{i} \wedge \alpha^{I}+\frac{1}{6} T_{I J K}(X) \alpha^{I} \wedge \alpha^{J} \wedge \alpha^{K}\right) .
$$

Here $X: \Sigma_{3} \rightarrow M$ are the embedding fields of a three-dimensional membrane worldvolume $\Sigma_{3}$ in spacetime $M, \alpha \in \Omega^{1}\left(\Sigma_{3}, X^{*} E\right)$ are auxilliary one-form fields on $\Sigma_{3}$ valued in the Courant algebroid $E$, and $\phi \in \Omega^{2}\left(\Sigma_{3}, X^{*} T^{*} M\right)$ are auxilliary two-form fields on $\Sigma_{3}$ valued in the cotangent bundle $T^{*} M$. In this paper we shall work only with the standard Courant algebroid, which in a suitable 
frame for the $\beta$-transformation symmetry is the generalized tangent bundle $E=C=T M \oplus T^{*} M$ with metric given by the natural dual pairing

$$
\left\langle Y_{1}+\lambda_{1}, Y_{2}+\lambda_{2}\right\rangle=\lambda_{2}\left(Y_{1}\right)+\lambda_{1}\left(Y_{2}\right)
$$

for vector fields $Y_{1}, Y_{2}$ and one-forms $\lambda_{1}, \lambda_{2}$ on $M$, and the anchor map is the projection $\rho: C \rightarrow$ $T M$. In the natural frame $\psi_{I}=\left(e_{i}, e^{i}\right)$ the $\mathrm{O}(d, d)$-invariant metric has only the non-vanishing components

$$
\left\langle e_{i}, e^{j}\right\rangle=\delta_{i}^{j}
$$

\subsection{Sigma-models for geometric fluxes}

Let us consider first how to apply the AKSZ sigma-model formalism to the case of geometric flux compactifications, focusing for definiteness on the $H$-space duality frame. The relevant algebroid is the standard Courant algebroid $C=T M \oplus T^{*} M$ twisted by a three-form $H$-flux $H=\frac{1}{6} H_{i j k}(x) \mathrm{d} x^{i} \wedge \mathrm{d} x^{j} \wedge \mathrm{d} x^{k}$; the twisting is accounted for by equipping $C$ with the $H$-twisted Courant-Dorfman bracket

$$
\left[Y_{1}+\lambda_{1}, Y_{2}, \lambda_{2}\right]_{H}:=\left[Y_{1}, Y_{2}\right]_{T M}+\mathscr{L}_{Y_{1}} \lambda_{2}-\mathscr{L}_{Y_{2}} \lambda_{1}-\frac{1}{2} \mathrm{~d}\left(\lambda_{2}\left(Y_{1}\right)-\lambda_{1}\left(Y_{2}\right)\right)+H\left(Y_{1}, Y_{2},-\right),
$$

where $\mathscr{L}_{Y}$ denotes the Lie derivative along the vector field $Y$. The only non-vanishing Lie brackets and 3-brackets evaluated on the natural frame are given by

$$
\left[e_{i}, e_{j}\right]_{H}=H_{i j k} e^{k} \quad, \quad\left[e_{i}, e_{j}, e_{k}\right]_{H}=H_{i j k} .
$$

Let us now substitute these structure maps into the general action (3.2), and denote $\alpha:=\left(\alpha^{i}, \xi_{i}\right)$ with $\left(\alpha^{i}\right) \in C^{\infty}\left(\Sigma_{3}, X^{*} T M\right)$ and $\left(\xi_{i}\right) \in C^{\infty}\left(\Sigma_{3}, X^{*} T^{*} M\right)$. In this way we arrive at the action for the topological membrane [55]

$$
S_{\mathrm{AKSZ}}^{(2)}=\int_{\Sigma_{3}}\left(\phi_{i} \wedge \mathrm{d} X^{i}+\alpha^{i} \wedge \mathrm{d} \xi_{i}-\phi_{i} \wedge \alpha^{i}+\frac{1}{6} H_{i j k}(X) \alpha^{i} \wedge \alpha^{j} \wedge \alpha^{k}\right) .
$$

For open membranes with boundary the worldsheet $\Sigma_{2}:=\partial \Sigma_{3} \neq \varnothing$, we can add a boundary term $\int_{\Sigma_{2}} \frac{1}{2} \Theta^{i j}(X) \xi_{i} \wedge \xi_{j}$ for a fixed bivector $\Theta \in C^{\infty}\left(M, \wedge^{2} T M\right)$ to get the action of the boundary/bulk open topological membrane [34]. Integrating out the two-form fields $\phi_{i}$ sets $\alpha^{i}=\mathrm{d} X^{i}$, and using Stokes' theorem we arrive finally at the AKSZ string action

$$
\widetilde{S}_{\mathrm{AKSZ}}^{(1)}=\int_{\Sigma_{2}}\left(\xi_{i} \wedge \mathrm{d} X^{i}+\frac{1}{2} \Theta^{i j}(X) \xi_{i} \wedge \xi_{j}\right)+\int_{\Sigma_{3}} \frac{1}{6} H_{i j k}(X) \mathrm{d} X^{i} \wedge \mathrm{d} X^{j} \wedge \mathrm{d} X^{k} .
$$

This is the action of the $H$-twisted Poisson sigma-model with target space $M$ [46]; the volume term here is completely analogous to a Wess-Zumino-Witten term. Consistency of the equations of motion now leads to the on-shell condition $[\Theta, \Theta]_{S}=\wedge^{3} \Theta^{\sharp}(H)$, where $\Theta^{\sharp}(H)$ is the natural way of contracting the three-form $H$ into a trivector using $\Theta$. This condition means that the bivector $\Theta$ now defines an $H$-twisted Poisson structure; in this case the Jacobi identity for bracket $\{f, g\}_{\Theta}=\Theta(\mathrm{d} f, \mathrm{~d} g)$ is violated, with the Jacobiator controlled by the trivector $[\Theta, \Theta]_{S}$, i.e., $\{f, g, h\}_{\Theta}=\bigwedge^{3} \Theta^{\sharp}(H)(\mathrm{d} f, \mathrm{~d} g, \mathrm{~d} h)$. This is precisely the sigma-model that governs the topological sector of open string dynamics in non-constant $B$-fields; in particular, its correlation functions reproduce the nonassociative star products of Kontsevich's deformation quantization of twisted Poisson structures [21]. 


\subsection{Sigma-models for non-geometric fluxes}

Now let us turn to the AKSZ sigma-model formalism appropriate to non-geometric flux compactifications. We shall see that, in contrast to the $H$-space sigma-models, the appropriate $R$-space theory really is a membrane sigma-model, not a string theory, which geometrizes the non-geometric $R$-flux background, analogously to the way in which M-theory geometrizes the nonperturbative dynamics of string theory. Whether or not these membranes are fundamental degrees of freedom and related to the M2-branes of M-theory is not clear at present. This question could be investigated by finding a suitable topological twisting of the supersymmetric worldvolume theory of an M2-brane. As the Courant sigma-model is the unique three-dimensional topological field theory susceptible to the Batalin-Vilkovisky formalism, it should then coincide with the topological sector of this worldvolume theory. Further evidence for this relation is provided by the fact that the noncommutative loop algebra from $\$ 2.4$ agrees with the noncommutativity induced on the boundaries of open M2branes ending on an M5-brane in a constant $C$-field background [57, 58], which can be interpreted as the noncommutative geometry experienced by closed strings in constant $H$-flux backgrounds; this perspective helps to connect the open and closed string points of view required below.

Again we start with the general Courant sigma-model (3.2) for the standard Courant algebroid $C=T M \oplus T^{*} M$, but this time twisted by a trivector flux $R=\frac{1}{6} R^{i j k}(x) \partial_{i} \wedge \partial_{j} \wedge \partial_{k}$. This twisting is described by the Roytenberg bracket

$$
\left[Y_{1}+\lambda_{1}, Y_{2}+\lambda_{2}\right]_{R}:=\left[Y_{1}, Y_{2}\right]_{T M}+R\left(\lambda_{1}, \lambda_{2},-\right)+\mathscr{L}_{Y_{1}} \lambda_{2}-\mathscr{L}_{Y_{2}} \lambda_{1}-\frac{1}{2} \mathrm{~d}\left(\lambda_{2}\left(Y_{1}\right)-\lambda_{1}\left(Y_{2}\right)\right),
$$

which when evaluated on the natural frame for $C$ yields the non-vanishing Lie brackets and 3brackets

$$
\left[e^{i}, e^{j}\right]_{R}=R^{i j k} e_{k} \quad, \quad\left[e^{i}, e^{j}, e^{k}\right]_{R}=R^{i j k}
$$

that are naturally dual to the bracket relations (3.3). Substituting these structure maps into the action (3.2), and integrating out the two-form field $\phi$ as previously yields the action

$$
S_{R}^{(2)}=\int_{\Sigma_{2}} \xi_{i} \wedge \mathrm{d} X^{i}+\int_{\Sigma_{3}} \frac{1}{6} R^{i j k}(X) \xi_{i} \wedge \xi_{j} \wedge \xi_{k}+\int_{\Sigma_{2}} \frac{1}{2} g^{i j}(X) \xi_{i} \wedge * \xi_{j},
$$

where $g$ is a Riemannian metric on $M$ and $*$ is the Hodge duality operator associated to a chosen metric on the worldsheet $\Sigma_{2}$. Here we have explicitly broken the topological symmetry of the sigma-model by adding a metric dependent term, in order to ensure that a non-vanishing $R$-flux is consistent with the equations of motion; although this may seem like a somewhat $a d$ hoc procedure at the classical level, similar metric dependences would appear anyway in the quantum action through gauge fixing terms.

The membrane sigma-model (3.4) has thus far been written for a general $R$-flux compactification. Let us now assume that $R^{i j k}$ and $g^{i j}$ are constant. The equation of motion for $X$ implies that $\xi$ is a closed one-form on $\Sigma_{2}$, and hence we can write $\xi_{i}=\mathrm{d} P_{i}$ for some section $P \in C^{\infty}\left(\Sigma_{2}, X^{*} T^{*} M\right)$; we do not include possible harmonic one-form contributions to this expression, as they would drop out of the final expressions below anyway. Using Stokes' theorem, the action then reduces to a pure boundary action, which we can linearize with auxiliary fields $\eta_{I}$ to get a generalized Poisson sigma-model

$$
S_{R}^{(2)}=\int_{\Sigma_{2}}\left(\eta_{I} \wedge \mathrm{d} X^{I}+\frac{1}{2} \Theta^{I J}(X) \eta_{I} \wedge \eta_{J}\right)+\int_{\Sigma_{2}} \frac{1}{2} G^{I J} \eta_{I} \wedge * \eta_{J}
$$


where $X^{I}=\left(X^{1}, \ldots, X^{d}, P_{1}, \ldots, P_{d}\right)$ is interpreted as the embedding of the string worldsheet $\Sigma_{2}$ in the cotangent bundle $T^{*} M$ and

$$
\Theta=\left(\begin{array}{cc}
R^{i j k} p_{k} & \delta^{i}{ }_{j} \\
-\delta_{i}{ }^{j} & 0
\end{array}\right) \quad, \quad G^{I J}=\left(\begin{array}{cc}
g^{i j} & 0 \\
0 & 0
\end{array}\right) .
$$

Thus the effective target space of the non-geometric string theory is the phase space of the spacetime $M$.

From this sigma-model perspective, the quantity $\Theta$ is an $H$-twisted Poisson bivector on phase space $T^{*} M$, with non-vanishing Schouten bracket

$$
\Pi:=[\Theta, \Theta]_{S}=\bigwedge^{3} \Theta^{\sharp}(H)
$$

where

$$
H=\mathrm{d} B \quad, \quad B=\frac{1}{6} R^{i j k} p_{k} \mathrm{~d} p_{i} \wedge \mathrm{d} p_{j}
$$

is the curvature of a $\mathrm{U}(1)$ gerbe on momentum space. It determines a noncommutative/nonassociative phase space with twisted Poisson brackets

$$
\left\{x^{I}, x^{J}\right\}_{\Theta}=\Theta^{I J}(x)
$$

whose quantization reproduces the closed string commutation relations (2.5). The corresponding Jacobiator

$$
\left\{x^{I}, x^{J}, x^{K}\right\}_{\Theta}:=\Pi\left(x^{I}, x^{J}, x^{K}\right)=\left(\begin{array}{cc}
R^{i j k} & 0 \\
0 & 0
\end{array}\right)
$$

quantizes the closed string 3-brackets (2.6).

\section{Quantization of $\boldsymbol{R}$-flux string vacua}

\subsection{Path integral quantization}

We would now like to compute correlation functions of suitable operators in the $R$-space sigma-model. Ultimately, one would like to do this directly at the level of the membrane sigmamodel, as this is what geometrizes the $R$-flux background. However, quantization of the topological membrane theory is extremely difficult, as even the gauge-fixed action is immensely complicated; part of the problem is that, in addition to the usual gauge symmetries, the AKSZ sigma-models in general contain higher Lie algebroid symmetries and so require ghosts-for-ghosts in addition to the usual ghost fields. Instead, we can exploit the hidden open string that is implicit in the open membrane formulation of the closed string theory in $R$-space. The multivaluedness of the closed string fields discussed in $\$ 2.4$ are implemented in the underlying orbifold conformal field theory via insertions of twist fields in correlators which create branch cuts on the worldsheet. By extending the closed string worldsheet to a membrane worldvolume $\Sigma_{3}$, the resulting branched surface can be interpreted as an open string worldsheet; see [52] for further details of the path integral description of this closed/open string duality. An alternative perspective would be to allow for singularities and assume that the closed string worldsheet is a surface with corners; in that case one could in 
principle deal with the boundary correlation functions that we discuss below, but such an approach seems technically cumbersome and we do not know how to proceed with this point of view.

From this open string perspective, suitable functional integrals in the generalized Poisson sigma-model then reproduce Kontsevich's graphical expansion for global deformation quantization [17]. The key quantities that emerge from these perturbative computations are the Kontsevich formality maps $U_{n}$, which take $n$ multivector fields $\mathscr{X}_{1}, \ldots, \mathscr{X}_{n}$ on $\mathscr{M}:=T^{*} M$ to a multidifferential operator

$$
U_{n}\left(\mathscr{X}_{1}, \ldots, \mathscr{X}_{n}\right)=\sum_{\Gamma \in G_{n}} w_{\Gamma} D_{\Gamma}\left(\mathscr{X}_{1}, \ldots, \mathscr{X}_{n}\right),
$$

where the sum is taken over all admissible diagrams $\Gamma$ and the weights $w_{\Gamma}$ of graphs are computed from certain integrals over geodesic angles in the hyperbolic upper half-plane regarded as the disk $\Sigma_{2}$. The geodesics represent derivatives emanating from the multivector fields which act on functions that are inserted on the boundary $\partial \Sigma_{2} \cong \mathbb{R}$ of the upper half-plane. For example, the action of the bivector $\Theta=\frac{1}{2} \Theta^{I J} \partial_{I} \wedge \partial_{J}$ is represented as

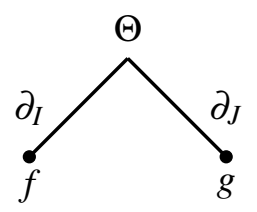

and it computes the star product

$$
f \star g=\sum_{n=0}^{\infty} \frac{(\mathrm{i} \hbar)^{n}}{n !} U_{n}(\Theta, \ldots, \Theta)(f, g)=: \Phi(\Theta)(f, g),
$$

while the action of the trivector $\Pi=\frac{1}{6} \Pi^{I J K} \partial_{I} \wedge \partial_{J} \wedge \partial_{K}=[\Theta, \Theta]_{S}$ is depicted by

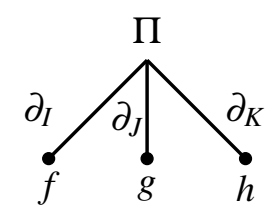

and it computes a 3-bracket

$$
[f, g, h]_{\star}=\sum_{n=0}^{\infty} \frac{(\mathrm{i} \hbar)^{n}}{n !} U_{n+1}(\Pi, \Theta, \ldots, \Theta)(f, g, h)=: \Phi(\Pi)(f, g, h),
$$

where $f, g, h \in C^{\infty}(\mathscr{M})$.

The maps $U_{n}$ define $L_{\infty}$-morphisms of differential graded Lie algebras relating Schouten brackets $[-,-]_{S}$ to Gerstenhaber brackets $[-,-]_{G}$, which are the natural extensions to multidifferential operators of the commutator bracket of differential operators; in particular, they satisfy formality conditions [47]. For example, the formality condition $[\Phi(\Theta), \star]_{G}=\mathrm{i} \hbar \Phi\left([\Theta, \Theta]_{S}\right)$ explicitly quantifies nonassociativity of the star product through the 3 -bracket as

$$
(f \star g) \star h-f \star(g \star h)=\frac{\hbar}{2 \mathrm{i}} \Phi(\Pi)(f, g, h)=\frac{\hbar}{2 \mathrm{i}}[f, g, h]_{\star} .
$$


The formality conditions also imply derivation properties. For instance, they map Hamiltonian vector fields to inner derivations of the star product and quasi-Poisson vector fields to differential operators which are derivations of the star product [52]. On the other hand, the formality condition $[\Phi(\Pi), \star]_{G}=\mathrm{i} \hbar \Phi\left([\Pi, \Theta]_{S}\right)$ encodes a quantum analogue of the Leibniz rule for the NambuPoisson structure $\{f, g, h\}_{\Pi}:=\Pi(\mathrm{d} f, \mathrm{~d} g, \mathrm{~d} h)$, which for the case at hand with constant $R$-flux implies

$$
[f \star g, h, k]_{\star}-[f, g \star h, k]_{\star}+[f, g, h \star k]_{\star}=f \star[g, h, k]_{\star}+[f, g, h]_{\star} \star k .
$$

Thus our construction also provides a means for quantizing Nambu-Poisson structures, which is a notoriously difficult unsolved problem in general (see e.g. [24] and references therein).

Explicit formulas can be obtained from the fact that all Kontsevich diagrams factorize and their weights can be expressed in terms of three diagrams (up to permutations), two involving the bivector field $\Theta$ and one involving the trivector field $\Pi$ [52]. This yields the dynamical nonassociative star-product

$$
f \star g=f \star_{p} g:=\cdot\left(\mathrm{e}^{\frac{\mathrm{i} \hbar}{2} R^{i j k} p_{k} \partial_{i} \otimes \partial_{j}} \mathrm{e}^{\frac{\mathrm{i} \hbar}{2}\left(\partial_{i} \otimes \tilde{\partial}^{i}-\tilde{\partial}^{i} \otimes \partial_{i}\right)}(f \otimes g)\right),
$$

where $\partial_{i}=\frac{\partial}{\partial x^{i}}$ and $\tilde{\partial}^{i}=\frac{\partial}{\partial p_{i}}$. Nonassociativity arises when the derivatives $\tilde{\partial}^{i}$ hit explicit momenta $p_{i}$ in the bidifferential operator defining the star product. By replacing the dynamical momentum variable $p$ with a constant momentum $\bar{p}$, we obtain an associative Moyal-Weyl type star-product $\bar{\star}:=\star_{\bar{p}}$. We can then express triple products in terms of a tridifferential operator as

$$
(f \star g) \star h=\left[\bar{\star}\left(\exp \left(\frac{\hbar^{2}}{4} R^{i j k} \partial_{i} \otimes \partial_{j} \otimes \partial_{k}\right)(f \otimes g \otimes h)\right)\right]_{\bar{p} \rightarrow p},
$$

where no ordering is required on the right-hand side because of associativity of the star product $\bar{\star}$, and the notation $\bar{p} \rightarrow p$ denotes the reinstatement of dynamical momentum. Nonassociativity is expressed through the explicit formula for the 3-bracket given by

$$
[f, g, h]_{\star}=\frac{4 \mathrm{i}}{\hbar}\left[\bar{\star}\left(\sinh \left(\frac{\hbar^{2}}{4} R^{i j k} \partial_{i} \otimes \partial_{j} \otimes \partial_{k}\right)(f \otimes g \otimes h)\right)\right]_{\bar{p} \rightarrow p} .
$$

This star product reproduces the fundamental phase space commutation relations

$$
\left[x^{i}, x^{j}\right]=\mathrm{i} \hbar R^{i j k} p_{k} \quad, \quad\left[x^{i}, p_{j}\right]=\mathrm{i} \hbar \delta_{j}^{i} \quad, \quad\left[p_{i}^{*}, p_{j}\right]=0,
$$

and it possesses the desired physical properties anticipated from on-shell closed string scattering amplitudes. For example, both 2-cyclicity and 3-cyclicity hold, i.e.,

$$
\begin{aligned}
\int_{\mathscr{M}} \mathrm{d}^{2 d} x f \star g & =\int_{\mathscr{M}} \mathrm{d}^{2 d} x g \star f=\int_{\mathscr{M}} \mathrm{d}^{2 d} x f g, \\
\int_{\mathscr{M}} \mathrm{d}^{2 d} x f \star(g \star h) & =\int_{\mathscr{M}} \mathrm{d}^{2 d} x(f \star g) \star h,
\end{aligned}
$$

showing that physical closed string states do not see noncommutativity or nonassociativity of the non-geometric flux background. In particular, when restricted to functions on configuration space $M$, the 3-product (4.3) reproduces the triproduct of [14] which was conjectured to reproduce offshell correlation functions of closed string tachyon vertex operators in $R$-space in a linearized conformal field theory analysis. The 2-cyclicity and 3-cyclicity properties are also the basis for a consistent formulation of nonassociative phase space quantum mechanics. 


\subsection{Seiberg-Witten maps and noncommutative gerbes}

In open string noncommutative gauge theory, the Seiberg-Witten map is an equivalence of (associative) star products $\star$ and $\star^{\prime}$ generated by a covariantizing map $\mathscr{D}$ which is a quantum analogue of Moser's lemma in symplectic geometry. Let $\Theta$ be a Poisson bivector, i.e., $[\Theta, \Theta]_{S}=0$, with dual two-form $B=\Theta^{-1}$, and let $\mathscr{L} \rightarrow \mathscr{M}$ be a line bundle with curvature $F=\mathrm{d} A$. Let $\rho$ be the flow generated by the vector field $\Theta(A,-)$. Then the map

$$
B \stackrel{\rho}{\longrightarrow} B+F
$$

is generated by a change of coordinates and quantizes to a map which sits in a commutative diagram $[41,42,43,44]$

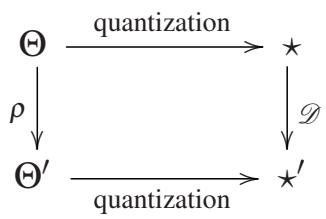

where $\Theta^{\prime}=\Theta(1+\hbar F \Theta)^{-1}$ is a new Poisson bivector, and $\mathscr{D}\left(f \star^{\prime} g\right)=\mathscr{D} f \star \mathscr{D} g$; the noncommutative gauge field $\hat{A}$ is defined by the covariant coordinates $\mathscr{D} x=: x+\hat{A}$ such that an ordinary gauge orbit of $A$ corresponds to a noncommutative gauge orbit of $\hat{A}$.

This construction can be used to realise the quantization of twisted Poisson structures in terms of noncommutative gerbes in the sense of [7]. Let $\Theta$ be a Poisson structure twisted by a closed three-form $H$, i.e., $[\Theta, \Theta]_{S}=\Lambda^{3} \Theta^{\sharp}(H)$. Let $\left\{U_{\alpha}\right\}$ be a good open covering of the manifold $\mathscr{M}$. Then on $U_{\alpha}$ the $H$-flux is generated by a locally defined $B$-field as $H=\mathrm{d} B_{\alpha}, B_{\alpha} \in \Omega^{2}\left(U_{\alpha}\right)$. On non-empty overlaps $U_{\alpha} \cap U_{\beta}$ the difference of potentials $B_{\beta}-B_{\alpha}$ is a closed two-form, hence $B_{\beta}-B_{\alpha}=F_{\alpha \beta}=\mathrm{d} A_{\alpha \beta}$ for local one-forms $A_{\alpha \beta}$ which determine $\mathrm{U}(1)$ gauge fields on a line bundle $\mathscr{L}_{\alpha \beta} \rightarrow U_{\alpha} \cap U_{\beta}$. The local $B$-fields can be used to locally untwist $\Theta$ to Poisson bivectors $\Theta_{\alpha}:=\Theta\left(1-\hbar B_{\alpha} \Theta\right)^{-1}$ on each patch $U_{\alpha}$. Then while the quantization of $\Theta$ yields a nonassociative star product $\star$, quantization of $\Theta_{\alpha}$ yields a family of local associative star products $\star_{\alpha}$ such that $\star_{\alpha}$ and $\star_{\beta}$ are equivalent by covariantizing maps $\mathscr{D}_{\alpha \beta}$ constructed from the curvatures $F_{\alpha \beta}$ on each non-empty overlap $U_{\alpha} \cap U_{\beta}$.

Now let us apply these general considerations to the construction of generalized SeibergWitten maps for non-geometric fluxes [52]. The $R$-twisted Poisson structure on phase space $\mathscr{M}=$ $T^{*} M$ is generated by the $\mathrm{U}(1)$ gerbe on momentum space with curvature (3.6); this is a trivial (but not flat) gerbe, so we can foliate $\mathscr{M}$ by surfaces of constant momentum and replace the open patch label $\alpha$ with the constant momentum vector $\bar{p}$. Then the relevant two-tensors are given by

$$
\Theta_{\bar{p}}=\left(\begin{array}{cc}
\hbar R^{i j k} \bar{p}_{k} \delta^{i}{ }_{j} \\
-\delta_{i}{ }^{j} & 0
\end{array}\right) \quad, \quad B_{\bar{p}}=\left(\begin{array}{lc}
0 & 0 \\
0 & R^{i j k}\left(p_{k}-\bar{p}_{k}\right)
\end{array}\right)
$$

where $\Theta_{\bar{p}}$ are Poisson bivectors which untwist the twisted Poisson bivector (3.5), and $H=\mathrm{d} B_{\bar{p}}=$ $\frac{1}{6} R^{i j k} \mathrm{~d} p_{i} \wedge \mathrm{d} p_{j} \wedge \mathrm{d} p_{k}$ is the curvature (3.6) of the trivial gerbe. The equivalence maps $\mathscr{D}_{\bar{p} \bar{p}^{\prime}}$ between associative star products $\bar{\star}$ and $\bar{\star}^{\prime}$ are generated by the gauge fields $A_{\bar{p} \bar{p}^{\prime}}=R^{i j k} p_{i}\left(\bar{p}_{k}-\bar{p}_{k}^{\prime}\right) \mathrm{d} p_{j}$ with curvature $F_{\bar{p} \bar{p}^{\prime}}=\frac{1}{2} R^{i j k}\left(\bar{p}_{k}-\bar{p}_{k}^{\prime}\right) \mathrm{d} p_{i} \wedge \mathrm{d} p_{j}$. In particular, for $\bar{p}=0$ we recover the canonical 
Moyal-Weyl star-product $\star_{0}$ on phase space. The map $\mathscr{D}_{\bar{p}}$ generated by $A_{\bar{p}}=R^{i j k} p_{i} \bar{p}_{k} \mathrm{~d} p_{j}$ from associative to nonassociative star products can be computed explicitly [52] and satisfies

$$
f \star g=\left[\mathscr{D}_{\bar{p}} f \star_{0} \mathscr{D}_{\bar{p}} g\right]_{\bar{p} \rightarrow p} .
$$

There is moreover an explicit nonassociative generalization of the Seiberg-Witten map suitable for the non-geometric backgrounds. A construction based directly on a twisted Poisson bivector $\Theta$ is usually spoiled by spurious terms involving the non-vanishing Schouten brackets $[\Theta, \Theta]_{S}$. However, in the present case such difficulties can be avoided by restricting to maps involving only gauge fields $A=\bar{a}^{i}(x, p) \mathrm{d} p_{i}$ which have no components along the configuration space $M$. There are two particular cases of interest in the context of this paper. Firstly, there are general coordinate transformations which are generated by the vector field $\Theta(A,-)=\bar{a}^{i}(x, p) \partial_{i}$ and are mapped to quantized diffeomorphisms; this lends some credibility to the hope that there is some notion of nonassociative gravity in non-geometric string backgrounds. Secondly, there are the NambuPoisson maps which are generated by $A=R\left(a_{2},-\right)$ for an arbitrary two-form $a_{2} \in \Omega^{2}(M)$, whose quantization leads to a higher gauge theory of quantized Nambu-Poisson tensor fields.

\subsection{3-cocycles and categorified Weyl quantization}

Let us now briefly explain some algebraic meanings behind the quantization of the twisted Poisson structure as alternative but equivalent quantizations to that obtained via the more analytical sigma-model approach. This can be elegantly understood through the origin of the generalized Poisson sigma-model as an AKSZ topological field theory with target space a Courant algebroid; in this way quantization can be cast as the problem of convolution quantization of Lie 2-algebras, which are categorifications of Lie algebras.

The reduction of the standard $R$-space Courant algebroid from before to the case where the base space $M$ is a point yields a quadratic Lie algebra $\mathfrak{h}$ with commutation relations

$$
\left[x^{i}, x^{j}\right]_{Q}=R^{i j k} \bar{p}_{k} \quad, \quad\left[x^{i}, \bar{p}_{j}\right]_{Q}=0=\left[\bar{p}_{i}, \bar{p}_{j}\right]_{Q}
$$

and an $\mathfrak{h}$-invariant $\mathrm{O}(d, d)$-symmetric inner product with non-vanishing values

$$
\left\langle x^{i}, \bar{p}_{j}\right\rangle=\delta_{j}^{i}
$$

These are just the commutation relations of a Heisenberg Lie algebra, and they mimick the closed string relations (2.4) in the $Q$-space duality frame; in particular, the Lie algebra $\mathfrak{h}$ can be regarded as a quantization of the Poisson structure $\Theta_{\bar{p}}$ from (4.6). This quadratic Lie algebra induces in the standard way a skeletal 2-term $L_{\infty}$-algebra

$$
V_{1}=\mathbb{R} \stackrel{0}{\rightarrow} V_{0}=\mathfrak{h}
$$

with classifying 3-cocycle $j: \mathfrak{h} \wedge \mathfrak{h} \wedge \mathfrak{h} \rightarrow \mathbb{R}$, in the Chevalley-Eilenberg cohomology of $\mathfrak{h}$ with values in the trivial representation, whose sole non-vanishing values are given by

$$
j\left(x^{i}, x^{j}, x^{k}\right)=\frac{1}{6}\left\langle\left[x^{i}, x^{j}\right]_{Q}, x^{k}\right\rangle+\text { cyclic }=R^{i j k} .
$$


This Lie 2-algebra can be integrated in the usual way to a Lie 2-group

$$
\mathscr{G}_{1}=G_{\mathfrak{h}} \times \mathrm{U}(1) \Longrightarrow \mathscr{G}_{0}=G_{\mathfrak{h}}
$$

with $G_{\mathfrak{h}}$ the Heisenberg group integrating $\mathfrak{h}$ via the exponential map, whose associator integrates $j$ and defines a 3-cocycle in the Chevalley-Eilenberg cohomology of $G_{\mathfrak{h}}$ with values in $\mathbb{R}$. The roles of 3-cocycles of Lie algebra and Lie group cohomology in the quantization of nonassociative $R$-space is elucidated in [9]. By computing convolution type products in this Lie 2-group, one can mimick the standard approach based on Weyl quantization in the associative setting of operator algebras (see e.g. [64] for a review) to rederive the nonassociative star products (4.2) for the phase space description of $R$-space; this algebraic approach is taken in [52,9].

\subsection{Quasi-Hopf cochain twist quantization}

There is yet another equivalent algebraic approach to the quantization of the $R$-flux background which emphasises the symmetries underlying the non-geometric flux compactification, and which provides a systematic means to obtain nonassociative deformations of geometry and gravity in $R$ space. This approach further illustrates in what sense nonassociative deformations really do grow naturally in the wild.

For this, let us first recall the associative setting of Hopf cocycle twist quantization [50]. A Drinfel'd twist for a Hopf algebra $H=H(\Delta, S, \varepsilon, \cdot)$ is an invertible counital element

$$
F=F_{(1)} \otimes F_{(2)} \in H \otimes H
$$

which satisfies the 2-cocycle condition

$$
(F \otimes 1) \Delta_{1} F=(1 \otimes F) \Delta_{2} F
$$

with $\Delta_{1}=\Delta \otimes 1$ and $\Delta_{2}=1 \otimes \Delta$. The twist $F$ can be used to map $H$ to a new Hopf algebra $H_{F}=$ $H_{F}\left(\Delta_{F}, S_{F}, \varepsilon, \cdot\right)$ with the same underlying algebraic structure but modified coalgebra structure; in particular, the new coproduct is given by

$$
\Delta_{F}=F \Delta F^{-1} .
$$

This construction quantizes any $H$-module algebra $A$ to a "braided-commutative" algebra $A_{F}$ with deformed product

$$
f \star g=\cdot\left(F^{-1}(f \otimes g)\right)=F_{(1)}^{-1} f \cdot F_{(2)}^{-1} g
$$

for $f, g \in A$; this deformation ensures that the action of the twisted Hopf algebra $H_{F}$ is compatible with the product on $A_{F}$, i.e., that $A_{F}$ is an $H_{F}$-module algebra.

Now let us relax the 2-cocycle condition and consider an arbitrary 2-cochain twist $F \in H \otimes H$. In that case $H_{F}$ is only a quasi-Hopf algebra, i.e., the coassociativity of the coproduct is violated in a controlled way

$$
\left(\Delta_{F}\right)_{2} \Delta_{F}=\phi\left(\Delta_{F}\right)_{1} \Delta_{F} \phi^{-1}
$$

by means of a multiplicative associator $\phi=\phi_{(1)} \otimes \phi_{(2)} \otimes \phi_{(3)} \in H \otimes H \otimes H$ which is a 3-cocycle obtained as the coboundary of the cochain twist via

$$
\phi=\partial^{*} F:=F_{23} \Delta_{2} F \Delta_{1} F^{-1} F_{12}^{-1}
$$


where $F_{23}=1 \otimes F_{(1)} \otimes F_{(2)}$ and $F_{12}=F_{(1)} \otimes F_{(2)} \otimes 1$. The notion of quasi-Hopf algebra was introduced in the early days of quantum groups by Drinfel'd when it was realised that many examples of quantum universal enveloping algebras satisfy only this weaker criterion. Now an $H$-module algebra $A$ gets quantized to a "quasi-associative" $H_{F}$-module algebra $A_{F}$ with

$$
(f \star g) \star h=\phi_{(1)} f \star\left(\phi_{(2)} g \star \phi_{(3)} h\right) .
$$

The power of this approach is its generality: Any algebraic entity can be quantized in this way, once a Hopf module structure and 2-cochain are chosen. For our applications we are interested in the specific example where $H=U(\mathfrak{g})$ is the enveloping Hopf algebra of a Lie algebra $\mathfrak{g}$ of symmetries acting on a manifold $\mathscr{M}$; then the algebra of functions $A=C^{\infty}(\mathscr{M})$ can be quantized in this way to a generically noncommutative and nonassociative algebra $A_{F}$. Similarly, the exterior algebra $\Omega^{\bullet}(\mathscr{M})$ of differential forms on $\mathscr{M}$ is quantized to $\Omega_{F}^{\bullet}(\mathscr{M})$, and so on for other geometrical structures.

This construction can be formalised into the notion of a twist quantization functor, i.e., it simultaneously deforms all $H$-covariant constructions as a functorial isomorphism

$$
\mathscr{Q}_{F}:{ }^{H} \mathfrak{M} \longrightarrow{ }^{{ }_{F}} \mathfrak{M}
$$

of braided monoidal categories of left $H$-modules ${ }^{H} \mathfrak{M}$ and left $H_{F}$-modules ${ }^{H_{F}} \mathfrak{M}$. In the monoidal category $H_{F} \mathfrak{M}$ the associator $\phi \in H \otimes H \otimes H$ induces non-trivial associativity isomorphisms $\Phi_{V, W, Z}$ : $(V \otimes W) \otimes Z \rightarrow V \otimes(W \otimes Z)$ defined by

$$
\Phi_{V, W, Z}((v \otimes w) \otimes z)=\phi_{(1)} v \otimes\left(\phi_{(2)} w \otimes \phi_{(3)} z\right) .
$$

The five-term 3-cocycle condition on $\phi$ implies that $\Phi$ obeys MacLane's pentagon relations; from the perspective of quantization of 3-brackets, the pentagon relations yield derivation properties such as (4.1). On the other hand, a braiding of the category ${ }_{F} \mathfrak{M}$ is provided by a quasi-triangular structure on the Hopf algebra and it induces non-trivial commutativity isomorphisms $\Psi_{V, W}: V \otimes$ $W \rightarrow W \otimes V$ given by

$$
\Psi_{V, W}(v \otimes w)=F_{(1)}^{-2} w \otimes F_{(2)}^{-2} v .
$$

From this perspective, both noncommutativity and nonassociativity are very natural features provided we work in the "right" category: While the algebra $A_{F}$ is noncommutative and nonassociative when normally considered as an object of the category of vector spaces, which has trivial braiding and associator, it is commutative and associative in the category ${ }_{F} \mathfrak{M}$.

Let us now apply these general considerations to obtain the cochain twist quantization of $R$ space [53]. For this, let $\mathfrak{g}$ be the nonabelian Lie algebra of phase space translations and Bopp shifts whose action on $C^{\infty}(\mathscr{M})$ is generated by the vector fields

$$
P_{i}=\partial_{i} \quad, \quad \tilde{P}^{i}=\tilde{\partial}^{i} \quad, \quad M_{i j}=p_{i} \partial_{j}-p_{j} \partial_{i} .
$$

For $\sigma^{i j}=-\sigma^{j i} \in \mathbb{R}$, the vector fields $\sigma^{i j} M_{i j}$ leave momenta fixed and act on position coordinates as the familiar non-local Bopp shifts $x^{i} \mapsto x^{i}+\sigma^{i j} p_{j}$ from quantum mechanics which mix positions with momenta. They define a quasi-Hopf deformation of the universal enveloping algebra $U(\mathfrak{g})$ by the cochain twist

$$
\mathscr{F}=\exp \left[-\frac{\mathrm{i} \hbar}{2}\left(\frac{1}{4} R^{i j k}\left(M_{i j} \otimes P_{k}-P_{i} \otimes M_{j k}\right)+P_{i} \otimes \tilde{P}^{i}-\tilde{P}^{i} \otimes P_{i}\right)\right],
$$


and the quantization functor on the category of quasi-Hopf module algebras generates nonassociative algebras through the associator

$$
\phi=\partial^{*} \mathscr{F}=\exp \left(\frac{\hbar^{2}}{2} R^{i j k} P_{i} \otimes P_{j} \otimes P_{k}\right) .
$$

The corresponding deformed product on the algebra $C^{\infty}(\mathscr{M})$ coincides with the star product (4.2), while the associator reproduces the triple product structure (4.3) and coincides with the 3-cocycle encountered before in a different context.

To illustrate the utility of this approach in constructing deformations of geometry, let us work out the corresponding nonassociative exterior differential calculus. We start with the usual exterior algebra complex $\left(\Omega^{\bullet}(\mathscr{M}), \wedge, \mathrm{d}\right)$ of differential forms on phase space, and assume that the exterior derivative $\mathrm{d}$ is equivariant under the covariant action of the enveloping Hopf algebra $H=U(\mathfrak{g})$. Then the action of $H$ on $\Omega^{\bullet}(\mathscr{M})$ is given by Lie derivatives $\mathscr{L}_{h}$ along the vector fields corresponding to elements $h \in H$; on differentials the only non-trivial actions are given by

$$
M_{i j} \mathrm{~d} x^{k}:=\mathscr{L}_{M_{i j}}\left(\mathrm{~d} x^{k}\right)=\delta_{j}^{k} \mathrm{~d} p_{i}-\delta_{i}^{k} \mathrm{~d} p_{j} .
$$

The deformed exterior product $\omega \wedge_{\star} \eta:=\wedge\left(F^{-1}(\omega \otimes \eta)\right)$ is generically noncommutative and nonassociative with the basic relations

$$
\begin{gathered}
\mathrm{d} x^{I} \wedge_{\star} \mathrm{d} x^{J}=-\mathrm{d} x^{J} \wedge_{\star} \mathrm{d} x^{I}=\mathrm{d} x^{I} \wedge \mathrm{d} x^{J}, \\
\left(\mathrm{~d} x^{I} \wedge_{\star} \mathrm{d} x^{J}\right) \wedge_{\star} \mathrm{d} x^{K}=\mathrm{d} x^{I} \wedge_{\star}\left(\mathrm{d} x^{J} \wedge_{\star} \mathrm{d} x^{K}\right) .
\end{gathered}
$$

By the equivariance condition, the exterior derivative $\mathrm{d}$ does not undergo any deformation as it is a morphism of the category ${ }^{H_{F}} \mathfrak{M}$. The action of $C^{\infty}(\mathscr{M})$ on $\Omega^{\bullet}(\mathscr{M})$ by pointwise multiplication of a form with a function is quantized to a deformed $A_{\mathscr{F}}$-bimodule structure whose only non-trivial relations are given by

$$
x^{i} \star \mathrm{d} x^{j}=\mathrm{d} x^{j} \star x^{i}+\frac{\mathrm{i} \hbar}{2} R^{i j k} \mathrm{~d} p_{k} .
$$

The nonassociative differential calculus also obeys the requisite physical properties of graded 2cyclicity

$$
\int_{\mathscr{M}} \omega \wedge_{\star} \eta=(-1)^{|\omega||\eta|} \int_{\mathscr{M}} \eta \wedge_{\star} \omega=\int_{\mathscr{M}} \omega \wedge \eta
$$

and graded 3-cyclicity

$$
\int_{\mathscr{M}}\left(\omega \wedge_{\star} \eta\right) \wedge_{\star} \lambda=\int_{\mathscr{M}} \omega \wedge_{\star}\left(\eta \wedge_{\star} \lambda\right)
$$

\subsection{Quantum mechanics with a 3-cocycle}

The commutation relations (2.5) capture the nonassociative geometry that arises in $R$-flux backgrounds and are heuristically expected to lead to novel uncertainty principles for spacetime positions. However, a rigorous derivation requires a formulation of quantum mechanics adapted to a nonassociative setting. This excludes all standard associative operator algebra approaches and is complicated further by the fact that our algebras are not of Jordan type, which is essentially the only case for which nonassociative aspects of quantum mechanics have been studied so far. A generalization of the phase space formulation of quantum mechanics, based on the nonassociative star product (4.2) with fundamental commutation relations (4.4), appears to be the most convenient 
approach. An essential ingredient for consistency are the 2-cyclicity and 3-cyclicity properties (4.5) of the star product with respect to integration. In the following we give a brief overview of nonassociative quantum mechanics and some of its consequences, refering to [53] for further details.

An observable $A$ in this approach is a real-valued function on $2 d$-dimensional phase space $\mathscr{M}$. More generally, operators are complex-valued functions and are multiplied together with the star product (4.2). Dynamics is implemented via Heisenberg-type time evolution equations

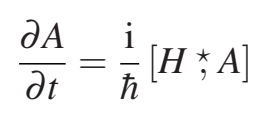

that look familiar, but in general are not derivations of the nonassociative star product operator algebra; similar evolution equations allow the study of the motion of a charge particle in a magnetic field with sources, i.e., $\nabla \cdot \boldsymbol{B} \neq 0$. States are characterized by normalized (complex-valued) functions $\psi_{\alpha}$ and statistical probabilities $\lambda_{\alpha}$ (for mixed states). Expectation values are computed via the phase space integral

$$
\langle A\rangle=\sum_{\alpha=1}^{n} \lambda_{\alpha} \int_{\mathscr{M}} \mathrm{d}^{2 d} x \psi_{\alpha}^{*} \star\left(A \star \psi_{\alpha}\right)=\int_{\mathscr{M}} \mathrm{d}^{2 d} x A S,
$$

and can be expressed as indicated in terms of a normalized real-valued state function

$$
S=\sum_{\alpha=1}^{n} \lambda_{\alpha} \psi_{\alpha} \star \psi_{\alpha}^{*}
$$

using (4.5). Given a state, we can define a semi-definite sesquilinear form for operators

$$
(A, B)=\sum_{\alpha=1}^{n} \lambda_{\alpha} \int_{\mathscr{M}} \mathrm{d}^{2 d} x\left(A \star \psi_{\alpha}\right)^{*}\left(B \star \psi_{\alpha}\right)
$$

that satisfies the Cauchy-Schwarz inequality

$$
|(A, B)|^{2} \leq(A, A)(B, B) .
$$

The inequality (4.7) is the basis for the derivation of uncertainty relations

$$
\Delta p_{i} \Delta p_{j} \geq 0 \quad, \quad \Delta x^{i} \Delta p_{j} \geq \frac{\hbar}{2} \delta^{i}{ }_{j} \quad, \quad \Delta x^{i} \Delta x^{j} \geq \frac{\hbar}{2}\left|R^{i j k}\left\langle p_{k}\right\rangle\right|,
$$

and encodes the concept of positivity of operators in our setting. Eigen-state functions $S$ of operators $A$ with eigenvalues $\lambda \in \mathbb{C}$ are defined as usual, i.e., $A \star S=\lambda S$, and observables $\left(A^{*}=A\right)$ have real eigenvalues. Due to nonassociativity, this fact is not straightforward, as $(A \star S) \star A^{*} \neq$ $A \star\left(S \star A^{*}\right)$; the proof requires 3-cyclicity (4.5). As in ordinary quantum mechanics, a pair of operators that do not commute cannot in general be measured simultaneously to arbitrary precision. A famous example are the limitations to position and momentum measurements imposed by the Heisenberg uncertainty principle. Only commuting operators have complete sets of common eigenstates. In nonassociative quantum mechanics, similar statements hold for triples of operators that do not associate. In particular any triple of coordinates $x^{i}, x^{j}, x^{k}$ that do not associate, i.e., with non-trivial $R$-flux $R^{i j k} \neq 0$, do not have any common eigenstate. The $R$-flux induced nonassociativity thus leads to a coarse-graining of spacetime with fundamental limitations to the determination 
of the exact locations of events. These new uncertainties are quantified by the non-zero expectation values of area and volume operators given by [53]

$$
\left\langle A^{i j}\right\rangle=\hbar R^{i j k}\left\langle p_{k}\right\rangle \quad, \quad\left\langle V^{i j k}\right\rangle=\frac{3}{2} \hbar^{2} R^{i j k} .
$$

\section{Acknowledgments}

R.J.S. thanks the organisors of the satellite conference for the opportunity to present these lectures and the warm hospitality during the meeting, and in particular Andrey Bytsenko for the invitation. The work of D.M. is supported by the Greek National Scholarship Foundation. The work of D.M. and R.J.S. was supported in part by the Consolidated Grant ST/J000310/1 from the U.K. Science and Technology Facilities Council. The work of P.S. was supported by the DFG RTG 1620 "Models of Gravity".

\section{References}

[1] G. Aldazabal, D. Marqués and C. Núñez, "Double field theory: A pedagogical review," Class. Quant. Grav. 30 (2013) 163001 [arXiv:1305.1907 [hep-th]].

[2] M. Alexandrov, M. Kontsevich, A. Schwartz and O. Zaboronsky, "The geometry of the master equation and topological quantum field theory,” Int. J. Mod. Phys. A 12 (1997) 1405-1430 [arXiv:hep-th/9502010].

[3] L. Álvarez-Gaumé, F. Meyer and M.A. Vázquez-Mozo, "Comments on noncommutative gravity," Nucl. Phys. B 753 (2006) 92-127 [arXiv:hep-th/0605113].

[4] D. Andriot and A. Betz, " $\beta$-supergravity: A ten-dimensional theory with non-geometric fluxes and its geometric framework," J. High Energy Phys. 1312 (2013) 083 [arXiv:1306.4381 [hep-th]].

[5] D. Andriot, M. Larfors, D. Lüst and P. Patalong, "(Non)commutative closed string on T-dual toroidal backgrounds," J. High Energy Phys. 1306 (2013) 021 [arXiv:1211.6437 [hep-th]].

[6] D. Andriot, O. Hohm, M. Larfors, D. Lüst and P. Patalong, "Non-geometric fluxes in supergravity and double field theory," Fortsch. Phys. 60 (2012) 1150-1186 [arXiv:1204.1979 [hep-th]].

[7] P. Aschieri, I. Bakovič, B. Jurčo and P. Schupp, "Noncommutative gerbes and deformation quantization,” J. Geom. Phys. 60 (2010) 1754-1761 [arXiv:hep-th/0206101].

[8] P. Aschieri, C. Blohmann, M. Dimitrijevic, F. Meyer, P. Schupp and J. Wess, "A gravity theory on noncommutative spaces," Class. Quant. Grav. 22 (2005) 3511-3532 [arXiv:hep-th/0504183].

[9] I. Bakas and D. Lüst, "3-cocycles, nonassociative star products and the magnetic paradigm of $R$-flux string vacua," arXiv:1309.3172 [hep-th].

[10] D.S. Berman and D.C. Thompson, “Duality symmetric string and M-theory,” arXiv:1306.2643 [hep-th].

[11] R. Blumenhagen and E. Plauschinn, "Nonassociative gravity in string theory?, J. Phys. A 44 (2011) 015401 [arXiv:1010.1263 [hep-th]].

[12] R. Blumenhagen, A. Deser, E. Plauschinn and F. Rennecke, "Non-geometric strings, symplectic gravity and differential geometry of Lie algebroids," J. High Energy Phys. 1302 (2013) 122 [arXiv:1211.0030 [hep-th]]. 
[13] R. Blumenhagen, M. Fuchs, F. Hassler, D. Lüst and R. Sun, "Nonassociative deformations of geometry in double field theory," arXiv:1312.0719 [hep-th].

[14] R. Blumenhagen, A. Deser, D. Lüst, E. Plauschinn and F. Rennecke, "Non-geometric fluxes, asymmetric strings and nonassociative geometry,” J. Phys. A 44 (2011) 385401 [arXiv:1106.0316 [hep-th]].

[15] P. Bouwknegt, K.C. Hannabuss and V. Mathai, "Nonassociative tori and applications to T-duality," Commun. Math. Phys. 264 (2006) 41-69 [arXiv:hep-th/0412092].

[16] J. Brodzki, V. Mathai, J.M. Rosenberg and R.J. Szabo, "Noncommutative correspondences, duality and D-branes in bivariant K-theory,” Adv. Theor. Math. Phys. 13 (2009) 497-552 [arXiv:0708.2648 [hep-th]].

[17] A.S. Cattaneo and G. Felder, "A path integral approach to the Kontsevich quantization formula," Commun. Math. Phys. 212 (2000) 591-611 [arXiv:math.QA/9902090].

[18] A. Chatzistavrakidis and L. Jonke, "Matrix theory origins of non-geometric fluxes," J. High Energy Phys. 1302 (2013) 040 [arXiv:1207.6412 [hep-th]].

[19] C.-S. Chu and P.-M. Ho, "Noncommutative open string and D-brane,” Nucl. Phys. B 550 (1999) 151-168 [arXiv:hep-th/9812219].

[20] C. Condeescu, I. Florakis and D. Lüst, “Asymmetric orbifolds, non-geometric fluxes and noncommutativity in closed string theory,” J. High Energy Phys. 1204 (2012) 121 [arXiv:1202.6366 [hep-th]].

[21] L. Cornalba and R. Schiappa, "Nonassociative star product deformations for D-brane worldvolumes in curved backgrounds," Commun. Math. Phys. 225 (2002) 33-66 [arXiv:hep-th/0101219].

[22] A. Dabholkar and C.M. Hull, “Duality twists, orbifolds and fluxes,” J. High Energy Phys. 0309 (2003) 054 [arXiv:hep-th/0210209].

[23] A. Dabholkar and C.M. Hull, "Generalised T-duality and non-geometric backgrounds," J. High Energy Phys. 0605 (2006) 009 [arXiv:hep-th/0512005].

[24] J. DeBellis, C. Saemann and R.J. Szabo, “Quantized Nambu-Poisson manifolds and n-Lie algebras," J. Math. Phys. 51 (2010) 122303 [arXiv:1001.3275 [hep-th]].

[25] M.R. Douglas and N.A. Nekrasov, “Noncommutative field theory,” Rev. Mod. Phys. 73 (2001) 977-1029 [arXiv:hep-th/0106048].

[26] I. Ellwood and A. Hashimoto, "Effective descriptions of branes on non-geometric tori," J. High Energy Phys. 0612 (2006) 025 [arXiv:hep-th/0607135].

[27] M. Graña, R. Minasian, M. Petrini and D. Waldram, “T-duality, generalized geometry and non-geometric backgrounds,” J. High Energy Phys. 0904 (2009) 075 [arXiv:0807.4527 [hep-th]].

[28] P. Grange and S. Schäfer-Nameki, "T-duality with $H$-flux: Noncommutativity, T-folds and $G \times G$ structure,” Nucl. Phys. B 770 (2007) 123-144 [arXiv:hep-th/0609084].

[29] N. Halmagyi, "Non-geometric backgrounds and the first order string sigma-model," arXiv:0906.2891 [hep-th].

[30] S. Hellerman, J. McGreevy and B. Williams, "Geometric constructions of non-geometric string theories,” J. High Energy Phys. 0401 (2004) 024 [arXiv:hep-th/0208174].

[31] M. Herbst, A. Kling and M. Kreuzer, "Star products from open strings in curved backgrounds," J. High Energy Phys. 0109 (2001) 014 [arXiv:hep-th/0106159]. 
[32] M. Herbst, A. Kling and M. Kreuzer, "Cyclicity of nonassociative products on D-branes," J. High Energy Phys. 0403 (2004) 003 [arXiv:hep-th/0312043].

[33] P.-M. Ho, “Making nonassociative algebra associative,” J. High Energy Phys. 0111 (2001) 026 [arXiv:hep-th/0103024].

[34] C. Hofman and J.-S. Park, “Topological open membranes,” arXiv:hep-th/0209148.

[35] O. Hohm, C.M. Hull and B. Zwiebach, "Background independent action for double field theory," J. High Energy Phys. 1007 (2010) 016 [arXiv:1003.5027 [hep-th]].

[36] O. Hohm, D. Lüst and B. Zwiebach, "The spacetime of double field theory: Review, remarks, and outlook," arXiv:1309.2977 [hep-th].

[37] C.M. Hull, “A geometry for non-geometric string backgrounds,” J. High Energy Phys. 0510 (2005) 065 [arXiv:hep-th/0406102].

[38] C.M. Hull and B. Zwiebach, “Double field theory," J. High Energy Phys. 0909 (2009) 099 [arXiv:0904.4664 [hep-th]].

[39] N. Ikeda, "Chern-Simons gauge theory coupled with BF theory,” Int. J. Mod. Phys. A 18 (2003) 2689-2702 [arXiv:hep-th/0203043].

[40] R. Jackiw, “3-cocycle in mathematics and physics,” Phys. Rev. Lett. 54 (1985) 159-162.

[41] B. Jurčo and P. Schupp, “Noncommutative Yang-Mills from equivalence of star products,” Eur. Phys. J. C 14 (2000) 367-370 [arXiv:hep-th/0001032].

[42] B. Jurčo, P. Schupp and J. Wess, "Noncommutative gauge theory for Poisson manifolds," Nucl. Phys. B 584 (2000) 784-794 [arXiv:hep-th/0005005].

[43] B. Jurčo, P. Schupp and J. Wess, "Nonabelian noncommutative gauge theory via noncommutative extra dimensions,” Nucl. Phys. B 604 (2001) 148-180 [arXiv:hep-th/0102129].

[44] B. Jurčo, P. Schupp and J. Wess, “Noncommutative line bundle and Morita equivalence,” Lett. Math. Phys. 61 (2002) 171-186 [arXiv:hep-th/0106110].

[45] S. Kachru, M.B. Schulz, P.K. Tripathy and S.P. Trivedi, "New supersymmetric string compactifications,” J. High Energy Phys. 0303 (2003) 061 [arXiv:hep-th/0211182].

[46] C. Klimčík and T. Strobl, “WZW-Poisson manifolds,” J. Geom. Phys. 43 (2002) 341-344 [arXiv:math.SG/0104189].

[47] M. Kontsevich, "Deformation quantization of Poisson manifolds," Lett. Math. Phys. 66 (2003) 157-216 [arXiv:q-alg/9709040].

[48] D. Lüst, “T-duality and closed string noncommutative (doubled) geometry," J. High Energy Phys. 1012 (2010) 084 [arXiv:1010.1361 [hep-th]].

[49] D. Lüst, "Twisted Poisson structures and noncommutative/nonassociative closed string geometry," Proc. Science CORFU 2011 (2011) 086 [arXiv:1205.0100 [hep-th]].

[50] S. Majid, Foundations of Quantum Group Theory (Cambridge University Press, 1995).

[51] V. Mathai and J.M. Rosenberg, "T-duality for torus bundles with $H$-fluxes via noncommutative topology," Commun. Math. Phys. 253 (2004) 705-721 [arXiv:hep-th/0401168].

[52] D. Mylonas, P. Schupp and R.J. Szabo, "Membrane sigma-models and quantization of non-geometric flux backgrounds,” J. High Energy Phys. 1209 (2012) 012 [arXiv:1207.0926 [hep-th]]. 
[53] D. Mylonas, P. Schupp and R.J. Szabo, "Non-geometric fluxes, quasi-Hopf twist deformations and nonassociative quantum mechanics," arXiv:1312.1621 [hep-th].

[54] Y. Nambu, “Generalized Hamiltonian dynamics,” Phys. Rev. D 7 (1973) 2405-2412.

[55] J.-S. Park, “Topological open p-branes,” in: Symplectic Geometry and Mirror Symmetry, eds. K. Fukaya, Y.-G. Oh, K. Ono and G. Tian (World Scientific, 2001) 311-384 [arXiv:hep-th/0012141].

[56] D. Roytenberg, "AKSZ-BV formalism and Courant algebroid-induced topological field theories," Lett. Math. Phys. 79 (2007) 143-159 [arXiv:hep-th/0608150].

[57] C. Saemann and R.J. Szabo, “Groupoid quantization of loop spaces,” Proc. Science CORFU 2011 (2011) 046 [arXiv:1203.5921 [hep-th]].

[58] C. Saemann and R.J. Szabo, "Groupoids, loop spaces and quantization of 2-plectic manifolds," Rev. Math. Phys. 25 (2013) 1330005 [arXiv:1211.0395 [hep-th]].

[59] P. Schaller and T. Strobl, "Poisson structure induced (topological) field theories," Mod. Phys. Lett. A 9 (1994) 3129-3136 [arXiv:hep-th/9405110].

[60] J. Scherk and J.H. Schwarz, "How to get masses from extra dimensions," Nucl. Phys. B 153 (1979) 61-88.

[61] V. Schomerus, “D-branes and deformation quantization,” J. High Energy Phys. 9906 (1999) 030 [arXiv:hep-th/9903205].

[62] N. Seiberg and E. Witten, "String theory and noncommutative geometry," J. High Energy Phys. 9909 (1999) 032 [arXiv:hep-th/9908142].

[63] J. Shelton, W. Taylor and B. Wecht, "Non-geometric flux compactifications," J. High Energy Phys. 0510 (2005) 085 [arXiv:hep-th/0508133].

[64] R.J. Szabo, “Quantum field theory on noncommutative spaces,” Phys. Rept. 378 (2003) 207-299 [arXiv:hep-th/0109162].

[65] R.J. Szabo, "Magnetic backgrounds and noncommutative field theory,” Int. J. Mod. Phys. A 19 (2004) 1837-1862 [arXiv:physics/0401142].

[66] R.J. Szabo, "Quantization of higher abelian gauge theory in generalized differential cohomology," Proc. Science ICMP 2012 (2012) 009 [arXiv:1209.2530 [hep-th]]. 